\title{
Closed surfaces with bounds on their Willmore energy
}

\author{
ERNST KUWERT AND REINER SCHÄTZLE
}

\begin{abstract}
The Willmore energy of a closed surface in $\mathbb{R}^{n}$ is the integral of its squared mean curvature, and is invariant under Möbius transformations of $\mathbb{R}^{n}$. We show that any torus in $\mathbb{R}^{3}$ with energy at most $8 \pi-\delta$ has a representative under the Möbius action for which the induced metric and a conformal metric of constant (zero) curvature are uniformly equivalent, with constants depending only on $\delta>0$. An analogous estimate is also obtained for closed, orientable surfaces of fixed genus $p \geq 1$ in $\mathbb{R}^{3}$ or $\mathbb{R}^{4}$, assuming suitable energy bounds which are sharp for $n=3$. Moreover, the conformal type is controlled in terms of the energy bounds.
\end{abstract}

Mathematics Subject Classification (2010): 53A05 (primary); 53A30, 53C21, 49Q15 (secondary).

\section{Introduction}

For an immersed surface $f: \Sigma \rightarrow \mathbb{R}^{n}$ the Willmore functional is defined as the integral

$$
\mathcal{W}(f)=\frac{1}{4} \int_{\Sigma}|\vec{H}|^{2} \mathrm{~d} \mu_{g}
$$

where $\vec{H}$ is the mean curvature vector, $g=f^{*} g_{\text {euc }}$ is the pull-back metric and $\mu_{g}$ is the induced area measure on $\Sigma$. The Gauß equation says that

$$
K=\frac{1}{2}\left(|\vec{H}|^{2}-|A|^{2}\right)=\frac{1}{4}|\vec{H}|^{2}-\frac{1}{2}\left|A^{\circ}\right|^{2},
$$

where $A_{i j}=A_{i j}^{\circ}+\frac{1}{2} \vec{H} g_{i j}$ is the vector-valued second fundamental form and $K$ is the sectional curvature of $g$. In the case when $\Sigma$ is a closed, orientable surface of genus $p$, the Gauß-Bonnet theorem therefore implies the identities

$$
\mathcal{W}(f)=\frac{1}{4} \int_{\Sigma}|A|^{2} \mathrm{~d} \mu_{g}+2 \pi(1-p)=\frac{1}{2} \int_{\Sigma}\left|A^{\circ}\right|^{2} \mathrm{~d} \mu_{g}+4 \pi(1-p) .
$$

Both authors were supported by the Deutsche Forschungsgemeinschaft via DFG Forschergruppe 469, and by the Centro di Ricerca Matematica Ennio De Giorgi during a visit in Pisa.

Received October 12, 2010; accepted in revised form March 10, 2011. 
We denote by $\beta_{p}^{n}$ the infimum of the Willmore functional among closed, orientable surfaces $f: \Sigma \rightarrow \mathbb{R}^{n}$ of genus $p$. It is well-known that $\beta_{0}^{n}=4 \pi$ with round spheres as unique minimizers. For $p \geq 1$ we have $\beta_{p}^{n}>4 \pi$ by the analysis of L. Simon [13]. We put

$$
\tilde{\beta}_{p}^{n}=\min \left\{4 \pi+\sum_{i=1}^{k}\left(\beta_{p_{i}}^{n}-4 \pi\right): 1 \leq p_{i}<p, \sum_{i=1}^{k} p_{i}=p\right\},
$$

where $\tilde{\beta}_{1}^{n}=\infty$, and define the constants

$$
\omega_{p}^{n}= \begin{cases}\min \left(8 \pi, \tilde{\beta}_{p}^{3}\right) & \text { for } n=3 \\ \min \left(8 \pi, \tilde{\beta}_{p}^{4}, \beta_{p}^{4}+\frac{8 \pi}{3}\right) & \text { for } n=4\end{cases}
$$

The main result of this paper is the following bilipschitz estimate. As the Willmore functional is invariant under the Möbius group of $\mathbb{R}^{n}$, i.e. under dilations and inversions, the choice of the Möbius transformation in the statement is essential.

Theorem 1.1. For $n=3,4$ and $p \geq 1$, let $\mathcal{C}(n, p, \delta)$ be the class of closed, orientable, genus $p$ surfaces $f: \Sigma \rightarrow \mathbb{R}^{n}$ satisfying $\mathcal{W}(f) \leq \omega_{p}^{n}-\delta$ for some $\delta>0$. Then for any $f \in \mathcal{C}(n, p, \delta)$ there is a Möbius transformation $\phi$ and a constant curvature metric $g_{0}$, such that the metric $g$ induced by $\phi \circ f$ satisfies

$$
g=e^{2 u} g_{0} \quad \text { where } \quad \max _{\Sigma}|u| \leq C(p, \delta)<\infty .
$$

We have $\beta_{p}^{n}<8 \pi$ as observed by Pinkall and independently Kusner, see for example [6], and $\beta_{p}^{n}<\tilde{\beta}_{p}^{n}$ from [1]. Thus $\mathcal{C}(n, p, \delta)$ is nonempty at least for small $\delta>0$. The stereographic projection of the Clifford torus into $\mathbb{R}^{3}$ has energy $2 \pi^{2}<8 \pi=\omega_{1}^{3}$ and is conjectured to be the minimizer for $p=1$, compare [11]. We remark that we would have $\omega_{p}^{n}=8 \pi$ once we knew that $\beta_{q}^{n} \geq 6 \pi$ for $1 \leq q<p$ and $\beta_{p}^{4} \geq 16 \pi / 3$ for $n=4$. It will be shown that our energy assumptions are sharp for $n=3$, that is the conclusion of the theorem fails if $\omega_{p}^{3}$ is replaced by any bigger constant. Combining the estimate in Theorem 4.2 with Mumford's compactness lemma we prove the following application.

Theorem 1.2. For $n \in\{3,4\}$ and $p \geq 1$, the conformal structures induced by immersions $f$ in $\mathcal{C}(n, p, \delta)$ are contained in a compact subset $K=K(p, \delta)$ of the moduli space.

In particular as $\omega_{1}^{4} \geq 20 \pi / 3$ we conclude $\mathcal{W}(f)>2 \pi^{2}$ for all tori $f: \Sigma \rightarrow$ $\mathbb{R}^{4}$ whose conformal structure is sufficiently degenerate. A straightforward second application of Theorem 1.1 is a compactness theorem, which will be stated in our forthcoming paper [8]. There the problem of minimizing the Willmore functional with prescribed conformal type is addressed. 
We now briefly summarize the contents of the paper. In Section 2 we review a version of the approximate graphical decomposition lemma on annuli, due to $\mathrm{L}$. Simon [13]. In Section 3 we present the global estimate of the conformal factor, under certain technical assumptions. The choice of the Möbius transformation is carried out in Section 4, and the proof of Theorem 4.1 is then completed by verifying the assumptions from Section 3. In Section 5 we discuss the bound for the conformal type and the optimality of the constant $\omega_{p}^{n}$. Our results rely on estimates for surfaces of the type of the plane due to S. Müller and V. Šverak [10]. The version needed is presented in the final Section 6.

ACKNOWLEDGEMENTS. The main ideas of this paper were developed during a joint visit at the Centro di Ricerca Matematica Ennio De Giorgi, Pisa. It is a pleasure to thank for the hospitality and the fruitful scientific atmosphere.

\section{Preliminaries}

Here we collect some results from the work of L. Simon [13], starting with consequences of the monotonicity identity. For a proper immersion $f: \Sigma \rightarrow B_{\varrho}(0) \subseteq$ $\mathbb{R}^{n}$ of an open surface $\Sigma$ and any $\sigma \in(0, \varrho)$, we have by (1.3) in [13] the bound

$$
\sigma^{-2} \mu\left(B_{\sigma}(0)\right) \leq C\left(\varrho^{-2} \mu\left(B_{\varrho}(0)\right)+\mathcal{W}\left(f, B_{\varrho}(0)\right)\right)
$$

where $\mu=f\left(\mu_{g}\right)$ is the pushforward area measure and

$$
\mathcal{W}\left(f, B_{\varrho}(0)\right)=\frac{1}{4} \int_{B_{\varrho}(0)}|\vec{H}|^{2} \mathrm{~d} \mu .
$$

We should really integrate over $f^{-1}\left(B_{\varrho}(0)\right)$ with respect to $\mu_{g}$, but the pullback is omitted for convenience; in fact the notation can be justified by considering $\mu$ as a 2 -varifold with square integrable weak mean curvature as in the appendix of [7]. If $\Sigma$ is compact without boundary we may let $\varrho \nearrow \infty$ in (2.1) to get

$$
\sigma^{-2} \mu\left(B_{\sigma}(0)\right) \leq C \mathcal{W}(f) \text { for all } \sigma>0 \text {. }
$$

Moreover, the multiplicity of the immersion at 0 is just the 2-density of $\mu$ and satisfies the Li-Yau inequality, see Theorem 6 in [9],

$$
\theta^{2}(\mu, 0) \leq \frac{1}{4 \pi} \mathcal{W}(f) .
$$

We will need the following version of the approximate graphical decomposition lemma, see Lemma 2.1 and [13, pages 312-315]. 
Lemma 2.1. For any $\Lambda<\infty$ there exist $\varepsilon_{0}=\varepsilon_{0}(n, \Lambda)>0$ and $C=C(n, \Lambda)<$ $\infty$ such that if $f: \Sigma \rightarrow B_{\varrho}(0) \subseteq \mathbb{R}^{n}$ is a proper immersion satisfying

$$
\mu\left(B_{\varrho}(0)-B_{\varrho / 2}(0)\right) \leq \Lambda \varrho^{2} \quad \text { and } \quad \int_{B_{\varrho}(0)-B_{\varrho / 2}(0)}|A|^{2} \mathrm{~d} \mu \leq \varepsilon^{2} \quad \text { for } \varepsilon<\varepsilon_{0},
$$

then the following statements hold:

(a) Denote by $A^{i}, i=1, \ldots, m$, those components of $f^{-1}\left(B_{7 \varrho / 8}(0)-B_{5 \varrho / 8}(0)\right)$ which extend to $\partial B_{9 \varrho / 16}(0)$. There exist compact subdiscs $P_{1}, \ldots, P_{N} \subseteq \Sigma$ with

$$
\sum_{j=1}^{N} \operatorname{diam} f\left(P_{j}\right)<C \varepsilon^{1 / 2} \varrho
$$

such that on each $A^{i}-\bigcup_{j=1}^{N} P_{j}$ the immersion is a $k_{i}$-valued graph for $k_{i} \in \mathbb{N}$, intersected with $B_{7 \varrho / 8}(0)-B_{5 \varrho / 8}(0)$, over some affine 2-plane. Furthermore

$$
M:=\sum_{i=1}^{m} k_{i} \leq C
$$

(b) There is a set $S \subseteq(5 \varrho / 8,7 \varrho / 8)$ of measure $\mathcal{L}^{1}(S)>3 \varrho / 16$, such that for $\sigma \in S$ the immersion is transversal to $\partial B_{\sigma}(0)$, and for each $\Gamma_{\sigma}^{i}:=A^{i} \cap f^{-1}\left(\partial B_{\sigma}(0)\right)$ we have

$$
\left|\int_{\Gamma_{\sigma}^{i}} \kappa_{g} \mathrm{~d} s-2 \pi k_{i}\right| \leq C \varepsilon^{\alpha} \quad \text { where } \alpha=\alpha(n)>0 .
$$

Furthermore, the restriction of $f$ to $A_{\sigma}^{i}:=A^{i} \cap f^{-1}\left(B_{\sigma}(0)\right)$ has a $C^{1,1}$ extension $\tilde{f}: \tilde{A}_{\sigma}^{i} \rightarrow \mathbb{R}^{n}$, where $\tilde{A}_{\sigma}^{i}$ is obtained by attaching a punctured disc $E_{\sigma}^{i}$ to $A_{\sigma}^{i}$ along $\Gamma_{\sigma}^{i}$, such that $\tilde{f}$ is a flat $k_{i}$-fold covering of an affine 2-plane $L_{i}$ outside $B_{2 \sigma}(0)$ and has curvature bounded by

$$
\int_{E_{\sigma}^{i}}|\tilde{A}|^{2} \mathrm{~d} \tilde{\mu} \leq C \varepsilon^{2}
$$

(c) If we assume in addition to (2.4) that

$$
\int_{B_{\varrho}(0)-B_{\varrho / 2}(0)} \frac{\left|x^{\perp}\right|^{2}}{|x|^{4}} \mathrm{~d} \mu(x)<\varepsilon^{2},
$$

where $\perp$ denotes the projection in the normal direction along the immersion, then $f^{-1}\left(B_{7 \varrho / 8}(0)-B_{5 \varrho / 8}(0)\right)=\bigcup_{i=1}^{m} A^{i}$ and we have the estimate

$$
\mu\left(B_{7 \varrho / 8}(0)-B_{5 \varrho / 8}(0)\right) \geq\left(1-C \varepsilon^{2 \alpha}\right) M \pi\left((7 \varrho / 8)^{2}-(5 \varrho / 8)^{2}\right) .
$$


If the assumptions of Lemma 2.1 hold with $\varrho / 2$ replaced by some $r \in(0, \varrho / 2]$, that is $\mu\left(B_{\varrho}(0)-B_{r}(0)\right) \leq \Lambda \varrho^{2}$ and

$$
\int_{B_{\varrho}(0)-B_{r}(0)}|A|^{2} \mathrm{~d} \mu, \int_{B_{\varrho}(0)-B_{r}(0)} \frac{\left|x^{\perp}\right|^{2}}{|x|^{4}} \mathrm{~d} \mu(x)<\varepsilon^{2},
$$

then by inequality (2.1) the assumptions of Lemma 2.1 are satisfied with $\varrho$ replaced by any $\sigma \in[2 r, \varrho]$. The resulting graphical decompositions have the same multiplicity $M$ by continuity. Choosing $\sigma_{v}=(5 / 7)^{v} \varrho$ and summing over the inequalities (2.9) we find

$$
\mu\left(B_{7 \varrho / 8}(0)-B_{5 r / 4}(0)\right) \geq\left(1-C \varepsilon^{2 \alpha}\right) M \pi\left((7 \varrho / 8)^{2}-(5 r / 4)^{2}\right) .
$$

The results in [13] are stated only for embedded surfaces, however they extend to immersions simply by considering a pertubation $f_{\lambda}=\left(f, \lambda f_{0}\right): \Sigma \rightarrow \mathbb{R}^{n} \times$ $\mathbb{R}^{3}$, where $f_{0}: \Sigma \rightarrow \mathbb{R}^{3}$ is some differentiable embedding. The $f_{\lambda}$ satisfy the assumptions of Lemma 2.1 for a slightly bigger constant $\Lambda$, hence they admit a graphical decomposition as stated over some 2 -planes in $\mathbb{R}^{n} \times \mathbb{R}^{3}$, which are almost horizontal for $\lambda$ sufficiently small. By slightly tilting the planes one obtains the desired almost graphical decomposition for the given immersion $f$, with power $\alpha=1 /(4 n+6)$ instead of $1 /(4 n-6)$ which is the constant in [13, Lemma 2.1].

\section{Oscillation estimates}

In this section we present the main PDE argument for the estimate of the conformal factor.

Theorem 3.1. Let $f: \Sigma \rightarrow \mathbb{R}^{n}, n=3$, 4, be an immersion of a closed, orientable surface $\Sigma$ of genus $p \geq 1$ with $\mathcal{W}(f) \leq \Lambda$. Assume that $f(\Sigma) \subseteq \bigcup_{k=1}^{K} B_{\varrho_{k} / 2}\left(x_{k}\right)$ with $\varrho_{l} \varrho_{k} \leq R$, such that for all $k=1, \ldots, K$ and some $\delta>0$ the following conditions hold:

$$
\begin{gathered}
\int_{B_{\varrho_{k}}\left(x_{k}\right)}|K| \mathrm{d} \mu<8 \pi-\delta \quad \text { for } n=3, \\
\int_{B_{\varrho_{k}}\left(x_{k}\right)}|K| \mathrm{d} \mu+\frac{1}{2} \int_{B_{\varrho_{k}}\left(x_{k}\right)}\left|A^{\circ}\right|^{2} \mathrm{~d} \mu<8 \pi-\delta \\
\left.\int_{B_{\varrho_{k}}\left(x_{k}\right)}\left|A^{\circ}\right|^{2} \mathrm{~d} \mu \quad \leq 8 \pi-C_{0} \varepsilon^{2}\right\} \\
\int_{B_{\varrho_{k}}\left(x_{k}\right)-B_{\ell_{k} / 2}\left(x_{k}\right)}|A|^{2} \mathrm{~d} \mu<\varepsilon^{2} .
\end{gathered}
$$

Denoting by $D_{\sigma}^{k, \alpha}, 1 \leq \alpha \leq m_{k}$, the components of $f^{-1}\left(B_{\sigma}\left(x_{k}\right)\right)$ which meet $\partial B_{9 \varrho_{k} / 16}\left(x_{k}\right)$, we further assume for all $\sigma \in\left[5 \varrho_{k} / 8,7 \varrho_{k} / 8\right]$ up to a set of measure 
at most $\varrho_{k} / 16$ that

$$
\int_{D_{\sigma}^{k, \alpha}} K \mathrm{~d} \mu_{g}>-2 \pi+\delta \quad \text { for all } \alpha=1, \ldots, m_{k} .
$$

Then for $\varepsilon \leq \varepsilon(\Lambda, \delta)$ and $C_{0} \geq C_{0}(\Lambda)$, there is a constant curvature metric $g_{0}=$ $e^{-2 u}$ g such that

$$
\max _{\Sigma}|u| \leq C(\Lambda, R, K, p, \delta)
$$

Proof. By rescaling we may assume $\mu_{g}(\Sigma)=1$. We take $g_{0}=e^{-2 u} g$ as the unique conformal, constant curvature metric also with $\mu_{g_{0}}(\Sigma)=1$, which means

$$
-\Delta_{g} u+K_{g_{0}} e^{-2 u}=K_{g} \quad \text { where } \quad K_{g_{0}}=\frac{2 \pi \chi(\Sigma)}{\mu_{g_{0}}(\Sigma)}=4 \pi(1-p) .
$$

Clearly the condition $\mu_{g_{0}}(\Sigma)=\mu_{g}(\Sigma)$ implies

$$
u(p)=0 \quad \text { for some } p \in \Sigma,
$$

and hence it suffices to prove the estimate

$$
\operatorname{osc}_{\Sigma} u \leq C(\Lambda, R, K, p, \delta) .
$$

The bound $\mathcal{W}(f) \leq \Lambda$ and the identity (1.2) imply

$$
\int_{\Sigma}|A|^{2} \mathrm{~d} \mu_{g} \leq C(\Lambda, p)
$$

and the Li-Yau inequality (2.2) yields

$$
\varrho^{-2} \mu\left(B_{\varrho}(x)\right) \leq C(\Lambda) \text { for all } B_{\varrho}(x) \subseteq \mathbb{R}^{n}
$$

The set of all $\sigma \in\left[5 \varrho_{k} / 8,7 \varrho_{k} / 8\right]$ satisfying both (3.4) and the inequality

$$
\int_{\partial B_{\sigma}\left(x_{k}\right)}|A|^{2} \mathrm{~d} s:=\int_{\partial\left[f^{-1}\left(B_{\sigma}\left(x_{k}\right)\right)\right]}|A|^{2} \mathrm{~d} s_{g} \leq 16 \varepsilon^{2} / \varrho_{k}
$$

has measure at least $\varrho_{k} / 8$. Thus we can choose $\sigma_{k}, \sigma_{k}^{\prime} \in\left[5 \varrho_{k} / 8,7 \varrho_{k} / 8\right]$ satisfying (3.4), (3.10) and the conclusions of Lemma 2.1(b), such that $\sigma_{k}-\sigma_{k}^{\prime}>\varrho_{k} / 16$. Since $f(\Sigma) \subseteq \bigcup_{k=1}^{K} B_{\varrho_{k} / 2}\left(x_{k}\right)$ we have

$$
\Sigma=\bigcup_{k, \alpha} D_{\sigma_{k}^{\prime}}^{k, \alpha}
$$

From the Gauß-Bonnet theorem and (3.4), we obtain for each component

$$
\int_{\partial D_{\sigma_{k}}^{k, \alpha}} \kappa_{g} \mathrm{~d} s_{g}=2 \pi \chi\left(D_{\sigma_{k}}^{k, \alpha}\right)-\int_{D_{\sigma_{k}}^{k, \alpha}} K \mathrm{~d} \mu_{g}<2 \pi\left(\chi\left(D_{\sigma_{k}}^{k, \alpha}\right)+1\right)-\delta .
$$


We conclude that each $D_{\sigma_{k}}^{k, \alpha}$ is a disc, and that the multiplicity of its boundary entering in (2.6) equals one, which means that all the graphs in Lemma 2.1(a) are singlevalued. Again by Lemma 2.1(b), we extend $\left.f\right|_{D_{\sigma_{k}}^{k, \alpha}}$ to an immersion $f_{k, \alpha}$ : $\Sigma_{k, \alpha} \rightarrow \mathbb{R}^{n}$ such that

$$
\int_{\Sigma_{k, \alpha}-D_{\sigma_{k}}^{k, \alpha}}\left|A_{f_{k, \alpha}}\right|^{2} \mathrm{~d} \mu_{f_{k, \alpha}} \leq C \varepsilon^{2} .
$$

Here $D_{\sigma_{k}}^{k, \alpha} \subseteq \Sigma_{k, \alpha} \cong \mathbb{R}^{2}$ and $f_{k, \alpha}$ is the standard embedding of a single plane outside $B_{2 \sigma_{k}}\left(x_{k}\right) \subseteq \mathbb{R}^{n}$, in particular $f_{k, \alpha}$ is complete. Now for $g_{k, \alpha}:=f_{k, \alpha}^{*} g_{\text {euc }}$ the Gauß-Bonnet theorem implies

$$
\int_{\Sigma_{k, \alpha}} K_{g_{k, \alpha}} \mathrm{d} \mu_{g_{k, \alpha}}=0 .
$$

By the uniformization theorem, we may assume that the diffeomorphism $\Sigma_{k, \alpha} \cong$ $\mathbb{R}^{2}$ is conformal, and write $g_{k, \alpha}=e^{2 u_{k, \alpha}} g_{\text {euc }}$ on $\Sigma_{k, \alpha} \cong \mathbb{R}^{2}$. From (3.1), (3.2) and (3.12), we get

$$
\begin{aligned}
& \int_{\Sigma_{k, \alpha}}\left|K_{g_{k, \alpha}}\right| \mathrm{d} \mu_{g_{k, \alpha}} \leq 8 \pi-\delta+C \varepsilon^{2} \text { for } n=3, \\
& \left.\begin{array}{c}
\int_{\Sigma_{k, \alpha}}\left|K_{g_{k, \alpha}}\right| \mathrm{d} \mu_{g_{k, \alpha}}+\frac{1}{2} \int_{\Sigma_{k, \alpha}}\left|A_{f_{k, \alpha}}^{\circ}\right|^{2} \mathrm{~d} \mu_{g_{k, \alpha}} \leq 8 \pi-\delta+C \varepsilon^{2} \\
\int_{\Sigma_{k, \alpha}}\left|A_{f_{k, \alpha}}^{\circ}\right|^{2} \mathrm{~d} \mu_{g_{k, \alpha}} \leq 8 \pi-C_{0} \varepsilon^{2}+C \varepsilon^{2}
\end{array}\right\} \quad \text { for } n=4 .
\end{aligned}
$$

Choosing $C \varepsilon^{2}<\delta / 2$ and $C_{0}>C$, this verifies the assumptions of Theorem 6.1, except that the parameter $\delta$ is replaced by $\delta / 2$. Thus we have

$$
-\Delta_{g_{k, \alpha}} u_{k, \alpha}=K_{g_{k, \alpha}} \text { in } \Sigma_{k, \alpha},
$$

where $u_{k, \alpha}$ satisfies the estimates, possibly after adding a suitable constant,

$$
\begin{aligned}
\left\|u_{k, \alpha}\right\|_{L^{\infty}\left(\Sigma_{k, \alpha}\right)},\left\|D u_{k, \alpha}\right\|_{L^{2}\left(\Sigma_{k, \alpha}\right)},\left\|D^{2} u_{k, \alpha}\right\|_{L^{1}\left(\Sigma_{k, \alpha}\right)} & \leq C(\delta) \int_{\Sigma_{k, \alpha}}\left|A_{f_{k, \alpha}}\right|^{2} \mathrm{~d} \mu_{g_{k, \alpha}} \\
& \leq C(\Lambda, p, \delta)
\end{aligned}
$$

Here the $L^{1}$ and $L^{2}$ norms on the left are with respect to the Euclidean metric on $\Sigma_{k, \alpha} \cong \mathbb{R}^{2}$, and we use (3.8) and (3.12) for the second inequality. As $f_{k, \alpha}$ and $f$ coincide on $D_{\sigma_{k}}^{k, \alpha}$, we have $g_{k, \alpha}=g$ on $D_{\sigma_{k}}^{k, \alpha}$, hence

$$
-\Delta_{g} u_{k, \alpha}=K_{g} \quad \text { in } D_{\sigma_{k}}^{k, \alpha}
$$

and by conformal invariance of the Dirichlet integral

$$
\left\|u_{k, \alpha}\right\|_{L^{\infty}\left(D_{\sigma_{k}}^{k, \alpha}\right)}, \int_{D_{\sigma_{k}}^{k, \alpha}}\left|D u_{k, \alpha}\right|_{g}^{2} \mathrm{~d} \mu_{g} \leq C(\Lambda, p, \delta) .
$$


Combining with (3.5) and as $g=g_{k, \alpha}=e^{2 u_{k, \alpha}} g_{\text {euc }}$ on $D_{\sigma_{k}}^{k, \alpha} \subseteq \Sigma_{k, \alpha} \cong \mathbb{R}^{2}$, we get

$$
-\Delta\left(u-u_{k, \alpha}\right)=-e^{2 u_{k, \alpha}} \Delta_{g}\left(u-u_{k, \alpha}\right)=-K_{g_{0}} e^{-2\left(u-u_{k, \alpha}\right)} \quad \text { in } D_{\sigma_{k}}^{k, \alpha},
$$

hence using $0 \leq-K_{g_{0}}=4 \pi(p-1)$ we conclude

$$
\left.\begin{array}{c}
-\Delta\left(u-u_{k, \alpha}\right) \geq 0, \\
-\Delta\left(u-u_{k, \alpha}\right)_{+} \leq C(p-1),
\end{array}\right\} \quad \text { in } D_{\sigma_{k}}^{k, \alpha} .
$$

Next we choose extrinsic cut-off functions $\gamma_{k} \in C_{0}^{2}\left(B_{5 \varrho_{k} / 8}\left(x_{k}\right)\right)$ with $0 \leq \gamma_{k} \leq 1$, $\gamma_{k}=1$ on $B_{\varrho_{k} / 2}\left(x_{k}\right)$ and $\left|D^{j} \gamma_{k}\right| \leq C \varrho_{k}^{-j}$ for $j=1,2$; we then put $\tilde{\eta}_{k, \alpha}:=\gamma_{k} \circ f$ on $D_{\sigma_{k}}^{k, \alpha}$ and $\tilde{\eta}_{k, \alpha}=0$ on $\Sigma-D_{\sigma_{k}}^{k, \alpha}$. Then $\tilde{\eta}:=\sum_{k, \alpha} \tilde{\eta}_{k, \alpha} \geq 1$ on $\Sigma$, as $f(\Sigma)$ is covered by the $B_{\varrho_{k} / 2}\left(x_{k}\right)$ for $k=1, \ldots, K$. We put $\eta_{k, \alpha}=\tilde{\eta}_{k, \alpha} / \tilde{\eta}$ and get

$$
\begin{gathered}
\operatorname{spt} \eta_{k, \alpha} \subseteq D_{\sigma_{k}}^{k, \alpha}, \\
\sum_{k, \alpha} \eta_{k, \alpha}=1, \\
\left|D \eta_{k, \alpha}\right|_{g} \leq C(R, K) \varrho_{k}^{-1}, \\
\left|D^{2} \eta_{k, \alpha}\right|_{g} \leq C(R, K)\left(\varrho_{k}^{-2}+\varrho_{k}^{-1}|A|\right) .
\end{gathered}
$$

Putting $\bar{u}:=\sum_{k, \alpha} \eta_{k, \alpha} u_{k, \alpha}$, we calculate from (3.5) and (3.13)

$$
-\Delta_{g}(u-\bar{u})=-K_{g_{0}} e^{-2 u}+2 \sum_{k, \alpha} D \eta_{k, \alpha} D u_{k, \alpha}+\sum_{k, \alpha} \Delta_{g} \eta_{k, \alpha} u_{k, \alpha}=: h
$$

and estimate by (3.8), (3.9), (3.14), recalling $K_{g_{0}}=4 \pi(1-p) \leq 0$ and $g_{0}=e^{-2 u} g$,

$$
\begin{aligned}
\int_{\Sigma}|h| \mathrm{d} \mu_{g} \leq & \int_{\Sigma}\left(-K_{g_{0}}\right) e^{-2 u} \mathrm{~d} \mu_{g} \\
& +C(R, K) \sum_{k, \alpha}\left(\varrho_{k}^{-2} \mu_{g}\left(D_{\sigma_{k}}^{k, \alpha}\right)\right)^{1 / 2}\left(\int_{D_{\sigma_{k}}^{k, \alpha}}\left|D u_{k, \alpha}\right|_{g}^{2} \mathrm{~d} \mu_{g}\right)^{1 / 2} \\
& +C(R, K) \sum_{k, \alpha}\left\|u_{k, \alpha}\right\|_{L^{\infty}\left(D_{\sigma_{k}}^{k, \alpha}\right)} \int_{D_{\sigma_{k}}^{k, \alpha}}\left(\varrho_{k}^{-2}+\varrho_{k}^{-1}|A|\right) \mathrm{d} \mu_{g} \\
\leq & C(\Lambda, R, K, p, \delta) .
\end{aligned}
$$

Furthermore

$$
\|\bar{u}\|_{L^{\infty}(\Sigma)}, \int_{\Sigma}|D \bar{u}|_{g}^{2} \mathrm{~d} \mu_{g} \leq C(\Lambda, R, K, p, \delta) .
$$

Multiplying (3.16) by $u-\bar{u}-\lambda$ where $\lambda \in \mathbb{R}$ is arbitrary, we obtain

$$
\begin{aligned}
\int_{\Sigma}|D(u-\bar{u})|_{g}^{2} \mathrm{~d} \mu_{g} & \leq \int_{\Sigma}|h||u-\bar{u}-\lambda| \mathrm{d} \mu_{g} \\
& \leq C(\Lambda, R, K, p, \delta)\|u-\bar{u}-\lambda\|_{L^{\infty}(\Sigma)} \\
& \leq C(\Lambda, R, K, p, \delta)\left(1+\|u-\lambda\|_{L^{\infty}(\Sigma)}\right),
\end{aligned}
$$


hence

$$
\begin{aligned}
\int_{\Sigma}|D u|_{g}^{2} \mathrm{~d} \mu_{g} & \leq 2 \int_{\Sigma}|D(u-\bar{u})|_{g}^{2} \mathrm{~d} \mu_{g}+2 \int_{\Sigma}|D \bar{u}|_{g}^{2} \mathrm{~d} \mu_{g} \\
& \leq C(\Lambda, R, K, p, \delta)\left(1+\operatorname{osc}_{\Sigma} u\right)
\end{aligned}
$$

Recalling the choice of $\sigma_{k}, \sigma_{k}^{\prime}$, we note $B_{\varrho_{k} / 16}^{g}(z) \subseteq D_{\sigma_{k}}^{k, \alpha}$ for $z \in D_{\sigma_{k}^{\prime}}^{k, \alpha}$, where $B_{\varrho}^{g}(z)$ is the geodesic ball with respect to $g$. Writing $B_{\varrho}^{2}(z)$ for the Euclidean coordinate disc using $\Sigma_{k, \alpha} \cong \mathbb{R}^{2}$, we see from (3.14) that $B_{2 c_{0} \varrho_{k}}^{2}(z) \subseteq D_{\sigma_{k}}^{k, \alpha}$ for $c_{0}=c_{0}(\Lambda, p, \delta)>0$ small enough. Now by (3.11) any $z \in \Sigma$ belongs to some $D_{\sigma_{l}^{\prime}}^{l, \beta}$, hence by (3.14) and $\mu_{g}(\Sigma)=1$

$$
\pi\left(c_{0} \varrho_{l}\right)^{2}=\mathcal{L}^{2}\left(B_{c_{0} \varrho_{l}}(z)\right) \leq C(\Lambda, p, \delta) \mu_{g}\left(D_{\sigma_{l}}^{l, \beta}\right) \leq C(\Lambda, p, \delta),
$$

hence $\varrho_{k} \leq C(\Lambda, R, p, \delta)$ for all $k$ since $\varrho_{k} / \varrho_{l} \leq R$ by assumption. Further by (3.9)

$$
1=\mu_{g}(\Sigma) \leq \sum_{k=1}^{K} \mu\left(B_{\varrho_{k} / 2}\left(x_{k}\right)\right) \leq C(\Lambda) K \max _{1 \leq k \leq K} \varrho_{k}^{2} .
$$

Using again $\varrho_{l} / \varrho_{k} \leq R$ we see that

$$
c_{0}(\Lambda, R, K, p) \leq \varrho_{k} \leq C(\Lambda, R, p, \delta) .
$$

Next, (3.17) and the Poincaré inequality show that, for appropriate $\lambda_{k, \alpha, z} \in \mathbb{R}$,

$$
\left(c_{0} \varrho_{k}\right)^{-1}\left\|u-\lambda_{k, \alpha, z}\right\|_{L^{2}\left(B_{c_{0} \varrho_{k}}^{2}(z)\right)} \leq C\|D u\|_{L^{2}\left(B_{c_{0} \varrho_{k}}^{2}(z)\right)} \leq C\left(1+\sqrt{\operatorname{osc}_{\Sigma} u}\right) .
$$

Select a maximal subset $\left\{z_{i}\right\}_{i \in I} \subseteq D_{\sigma_{k}^{\prime}}^{k, \alpha}$ with $B_{c_{0} \varrho_{k} / 4}^{2}\left(z_{i}\right)$ pairwise disjoint, whence the $B_{c_{0} \varrho_{k} / 2}^{2}\left(z_{i}\right), i \in I$, cover $D_{\sigma_{k}^{\prime}}^{k, l}$. As the $D_{\sigma_{k}}^{k, \alpha} \supseteq B_{c_{0} \varrho_{k} / 4}^{2}\left(z_{i}\right)$ are pairwise disjoint, we estimate the cardinality of $I$ by

$$
\begin{aligned}
\operatorname{card}(I) \pi\left(c_{0} \varrho_{k} / 4\right)^{2} & \leq \mathcal{L}^{2}\left(D_{\sigma_{k}}^{k, \alpha}\right) \leq C(\Lambda, p, \delta) \mu_{g}\left(D_{\sigma_{k}}^{k, \alpha}\right) \\
& \leq C(\Lambda, p, \delta) \mu\left(B_{\varrho_{k}}\left(x_{k}\right)\right) \leq C(\Lambda, p, \delta) \varrho_{k}^{2},
\end{aligned}
$$

as $g=e^{2 u_{k, \alpha}} g_{\text {euc }}$, using (3.14) and (3.9), hence

$$
\operatorname{card}(I) \leq C(\Lambda, p, \delta)
$$

If $B_{c_{0} \varrho_{k} / 2}^{2}\left(z_{i}\right) \cap B_{c_{0} \varrho_{k} / 2}^{2}\left(z_{j}\right) \neq \emptyset$, then $\mathcal{L}^{2}\left(B_{c_{0} \varrho_{k}}^{2}\left(z_{i}\right) \cap B_{c_{0} \varrho_{k}}^{2}\left(z_{j}\right)\right) \geq \pi\left(c_{0} \varrho_{k} / 2\right)^{2}$ and

$$
\begin{aligned}
\left|\lambda_{k, \alpha, z_{i}}-\lambda_{k, \alpha, z_{j}}\right| & \leq C\left(c_{0} \varrho_{k}\right)^{-1}\left(\left\|u-\lambda_{k, \alpha, z_{i}}\right\|_{L^{2}\left(B_{c_{0} \varrho_{k}}^{2}\left(z_{i}\right)\right)}+\left\|u-\lambda_{k, \alpha, z_{j}}\right\|_{L^{2}\left(B_{c_{0} \varrho_{k}}^{2}\left(z_{j}\right)\right)}\right) \\
& \leq C(\Lambda, K, p, \delta)\left(1+\sqrt{\operatorname{osc}_{\Sigma} u}\right) .
\end{aligned}
$$


As $D_{\sigma_{k}^{\prime}}^{k, \alpha}$ is connected and covered by the $B_{c_{0} \varrho_{k} / 2}^{2}\left(z_{i}\right)$, we find for $i, j \in I$ a chain $B_{c_{0} \varrho_{k} / 2}^{2}\left(z_{i_{v}}\right), v=1, \ldots, N$, with $N \leq \operatorname{card}(I)$ and such that neighboring discs intersect. Thus

$$
\left|\lambda_{k, \alpha, z_{i}}-\lambda_{k, \alpha, z_{j}}\right| \leq C(\Lambda, K, p, \delta)\left(1+\sqrt{\operatorname{osc}_{\Sigma} u}\right) \quad \forall i, j \in I .
$$

Therefore there exists a $\lambda_{k, \alpha} \in \mathbb{R}$ such that

$$
\varrho_{k}^{-1}\left\|u-\lambda_{k, \alpha}\right\|_{L^{2}\left(D_{\sigma_{k}^{\prime}}^{k, \alpha} ; g\right)} \leq C(\Lambda, K, p, \delta)\left(1+\sqrt{\operatorname{osc}_{\Sigma} u}\right) .
$$

The sets $B_{\varrho_{k} / 2}^{k, \alpha}:=D_{\sigma_{k}^{\prime}}^{k, \alpha} \cap f^{-1}\left(B_{\varrho_{k} / 2}\left(x_{k}\right)\right)$ form an open cover of $\Sigma$. Moreover if $z \in B_{\varrho_{k} / 2}^{k, \alpha} \cap B_{\varrho_{l} / 2}^{l, \beta}$ where $\varrho_{k} \leq \varrho_{l}$, then we obtain as above $B_{\varrho_{k} / 8}^{g}(z) \subseteq D_{\sigma_{k}^{\prime}}^{k, \alpha} \cap D_{\sigma_{l}^{\prime}}^{l, \beta}$, using $\sigma_{k}^{\prime} \geq 5 \varrho_{k} / 8, \sigma_{l}^{\prime} \geq 5 \varrho_{l} / 8$, and

$$
\mu_{g}\left(D_{\sigma_{k}^{\prime}}^{k, \alpha} \cap D_{\sigma_{l}^{\prime}}^{l, \beta}\right) \geq \mu_{g}\left(B_{\varrho_{k} / 8}^{g}(z)\right) \geq c_{0}(\Lambda, p, \delta) \mathcal{L}^{2}\left(B_{c_{0} \varrho_{k}}^{2}(z)\right) \geq c_{0}(\Lambda, p, \delta) \varrho_{k}^{2} .
$$

This yields

$$
\begin{aligned}
\left|\lambda_{k, \alpha}-\lambda_{l, \beta}\right| & \leq\left(c_{0} \varrho_{k}\right)^{-1}\left\|\lambda_{k, \alpha}-\lambda_{l, \beta}\right\|_{L^{2}\left(D_{\sigma_{k}^{\prime}}^{k, \alpha} \cap D_{\sigma_{l}^{\prime}}^{l, \beta} ; g\right)} \\
& \leq\left(c_{0} \varrho_{k}\right)^{-1}\left(\left\|u-\lambda_{k, \alpha}\right\|_{L^{2}\left(D_{\sigma_{k}^{\prime}}^{k, \alpha} ; g\right)}+\left\|u-\lambda_{l, \beta}\right\|_{L^{2}\left(D_{\sigma_{l}^{\prime}}^{l, \beta} ; g\right)}\right) \\
& \leq C(\Lambda, R, K, p, \delta)\left(1+\sqrt{\left.\operatorname{osc}_{\Sigma} u\right)},\right.
\end{aligned}
$$

as $\varrho_{l} / \varrho_{k} \leq R$ by assumption. Again by connectedness of $\Sigma$ there is a $\lambda \in \mathbb{R}$

$$
\|u-\lambda\|_{L^{2}(\Sigma ; g)} \leq C(\Lambda, R, K, p, \delta)\left(1+\sqrt{\operatorname{osc}_{\Sigma} u}\right) \underset{1 \leq k \leq K}{\max } \varrho_{k} .
$$

Next choose $z_{0} \in \Sigma$ with $u\left(z_{0}\right)=\min _{\Sigma} u$. Then $z_{0} \in B_{\varrho_{k} / 2}^{k, \alpha}$ for some $k, \alpha$. By (3.14) and (3.15) we have, as $B_{2 c_{0} \varrho_{k}}^{2}\left(z_{0}\right) \subseteq D_{\sigma_{k}}^{k, \alpha}$, the estimate $u-u_{k, \alpha} \geq$ $\min _{\Sigma} u-C(\Lambda, p, \delta)=: \bar{\lambda}$, and conclude from the weak Harnack inequality, see [3] Theorem 8.18,

$$
\left(c_{0} \varrho_{k}\right)^{-1}\left\|u-u_{k, \alpha}-\bar{\lambda}\right\|_{L^{2}\left(B_{c_{0} \varrho_{k}}^{2}\left(z_{0}\right)\right)} \leq C \inf _{B_{c_{0} \varrho_{k}}^{2}\left(z_{0}\right)}\left(u-u_{k, \alpha}-\bar{\lambda}\right) .
$$

Hence from $u\left(z_{0}\right)=\min _{\Sigma} u$ we see that

$$
\left(c_{0} \varrho_{k}\right)^{-1}\left\|u-\min _{\Sigma} u\right\|_{L^{2}\left(B_{c_{0} \varrho_{k}}^{2}\left(z_{0}\right)\right)} \leq C(\Lambda, p, \delta) .
$$

Then

$$
\left|\min _{\Sigma} u-\lambda\right| \leq C(\Lambda, R, K, p, \delta)\left(1+\sqrt{\operatorname{osc}_{\Sigma} u}\right) \max _{1 \leq k \leq K} \varrho_{k}
$$


by (3.19), and we conclude

$$
\left\|u-\min _{\Sigma} u\right\|_{L^{2}(\Sigma, g)} \leq C(\Lambda, R, K, p, \delta)\left(1+\sqrt{\operatorname{osc}_{\Sigma} u}\right) \max _{1 \leq k \leq K} \varrho_{k} .
$$

Now $\min _{\Sigma} u \leq 0$ by (3.6). Employing the mean value inequality, see [3] Theorem 2.1, we obtain from (3.15) for $z \in D_{\sigma_{k}^{\prime}}^{k, \alpha}$

$\left\|\left(u-u_{k, \alpha}\right)_{+}\right\|_{L^{\infty}\left(B_{c_{0} \varrho_{k}}^{2}(z)\right)} \leq C\left(c_{0} \varrho_{k}\right)^{-1}\left\|\left(u-u_{k, \alpha}\right)_{+}\right\|_{L^{2}\left(B_{2 c_{0} \varrho_{k}}^{2}(z)\right)}+C\left(c_{0} \varrho_{k}\right)^{2}(p-1)$.

Combining (3.14), (3.18), (3.20) and $\min _{\Sigma} u \leq 0$ implies

$$
\begin{aligned}
\max _{\Sigma} u & \leq C(\Lambda, p, \delta) \varrho_{k}^{-1}\left\|u-\min _{\Sigma} u\right\|_{L^{2}(\Sigma ; g)}+C(\Lambda, p, \delta)+C\left(c_{0} \varrho_{k}\right)^{2}(p-1) \\
& \leq C(\Lambda, R, K, p, \delta)\left(1+\sqrt{\operatorname{osc}_{\Sigma} u}\right) \\
& \leq C(\Lambda, R, K, p, \delta)+\frac{1}{2} \max _{\Sigma} u-\frac{1}{2} \min _{\Sigma} u,
\end{aligned}
$$

hence $\max _{\Sigma} u \leq C(\Lambda, R, K, p, \delta)+\left|\min _{\Sigma} u\right|$, and

$$
\operatorname{osc}_{\Sigma} u \leq C(\Lambda, R, K, p, \delta)+2\left|\min _{\Sigma} u\right| \text {. }
$$

Next we define $A=\left\{x \in \Sigma: u(x) \leq \min _{\Sigma} u / 2\right\}$. As $u-\min _{\Sigma} u \geq\left|\min _{\Sigma} u\right| / 2$ on $\Sigma-A$, we get from (3.18), (3.20) and (3.21)

$$
\begin{aligned}
\frac{1}{2}\left|\min _{\Sigma} u\right| \mu_{g}(\Sigma-A) & \leq \int_{\Sigma}\left(u-\min _{\Sigma} u\right) \mathrm{d} \mu_{g} \\
& \leq C(\Lambda, R, K, p, \delta)\left(1+\sqrt{\operatorname{osc}_{\Sigma} u}\right) \\
& \leq C(\Lambda, R, K, p, \delta)\left(1+\sqrt{\left|\min _{\Sigma} u\right|}\right) .
\end{aligned}
$$

Thus for $\left|\min _{\Sigma} u\right| \gg C(\Lambda, R, K, p, \delta)$ we estimate

$$
\mu_{g}(\Sigma-A) \leq \frac{C(\Lambda, R, K, p, \delta)\left(1+\sqrt{\left|\min _{\Sigma} u\right|}\right)}{\left|\min _{\Sigma} u\right|} \leq \frac{1}{2} .
$$

As both $g$ and $g_{0}=e^{-2 u} g$ have unit area, this yields $\mu_{g}(A) \geq 1 / 2$ and

$$
1 \geq \int_{A} e^{-2 u} \mathrm{~d} \mu_{g} \geq \mu_{g}(A) e^{-\min _{\Sigma} u} \geq \frac{1}{2} e^{\left|\min _{\Sigma} u\right|} .
$$

We conclude that $\left|\min _{\Sigma} u\right| \leq C(\Lambda, R, K, p, \delta)$, and hence (3.7) follows from (3.21), and the theorem is proved.

Inspecting the proof, we see that instead of (3.4) we could require directly that each component $D_{\sigma}^{k, \alpha}$ is a disc and $\left.f\right|_{\partial D_{\sigma}^{k, \alpha}}$ is a single, nearly flat circle, for all $\sigma \in$ ] $\varrho_{k} / 8,7 \varrho_{k} / 8$ [ up to a set of measure $\varrho_{k} / 16$. We also remark that the assumptions (3.1)-(3.4) are trivially implied by the single condition

$$
\int_{B_{\varrho_{k}}\left(x_{k}\right)}|A|^{2} \mathrm{~d} \mu<\varepsilon^{2} \quad \text { for all } k=1, \ldots, K .
$$

In fact, the estimate of the conformal factor can then be shown in any codimension. 


\section{Estimation modulo the Möbius group}

It will be essential in Theorem 4.2 to pass to a good representative under the action of the Möbius group. The following lemma yields the desired Möbius transformation.

Lemma 4.1. Let $f: \Sigma \rightarrow \mathbb{R}^{n}$ be an immersion of a closed surface $\Sigma$, with conformally invariant energy $\int_{\Sigma}\left|A^{\circ}\right|^{2} \mathrm{~d} \mu=:$ E. Then there exists a Möbius transformation $\phi$ such that $\tilde{f}=\phi \circ f$ satisfies $\tilde{f}(\Sigma) \subseteq B_{1}(0)$ and

$$
\int_{B_{\varrho_{0}}(x)}\left|\tilde{A}^{\circ}\right|^{2} \mathrm{~d} \tilde{\mu} \leq E / 2 \quad \text { for all } x \in \mathbb{R}^{n} \text {, where } \varrho_{0}=\varrho_{0}(n, E)>0 .
$$

Proof. By a dilation we may assume that for all $x \in \mathbb{R}^{n}$ and some $x_{0} \in \mathbb{R}^{n}$ we have

$$
\int_{B_{1}(x)}\left|A^{\circ}\right|^{2} \mathrm{~d} \mu \leq E / 2 \leq \int_{\overline{B_{1}\left(x_{0}\right)}}\left|A^{\circ}\right|^{2} \mathrm{~d} \mu .
$$

From (1.2) we see that the total Willmore energy of $f$ is bounded by

$$
\mathcal{W}(f) \leq E / 2+4 \pi .
$$

We now prove by area comparison arguments that there is a point $x \in \mathbb{R}^{n}$ satisfying

$$
\overline{B_{1}(x)} \cap f(\Sigma)=\emptyset \quad \text { and } \quad\left|x-x_{0}\right| \leq C(n, E) .
$$

The Li-Yau-inequality as in (2.2) yields the upper bound

$$
r^{-2} \mu\left(B_{r}\left(x_{0}\right)\right) \leq C(E) \quad \text { for any } r>0,
$$

while $\varrho=1$ and $\sigma \searrow 0$ in (2.1) yields

$$
\mu\left(B_{1}(x)\right)+\mathcal{W}\left(f, B_{1}(x)\right) \geq c>0 \text { for any } x \in f(\Sigma) .
$$

For $R>0$ to be chosen, let $B_{2}\left(x_{j}\right), j=1, \ldots, N$, be a maximal disjoint collection of 2-balls with centers $x_{j} \in B_{R}\left(x_{0}\right)$. As the balls $B_{4}\left(x_{j}\right)$ cover $B_{R}\left(x_{0}\right)$ we have $N \geq R^{n} / 4^{n}$. If $f(\Sigma) \cap \overline{B_{1}\left(x_{j}\right)} \neq \emptyset$ for all $j$, then (4.6), (4.5) and (4.3) imply

$$
c N \leq \sum_{j=1}^{N}\left(\mu\left(B_{2}\left(x_{j}\right)\right)+\mathcal{W}\left(f, B_{2}\left(x_{j}\right)\right)\right) \leq C(E)\left(R^{2}+1\right),
$$

thus $R \leq C(n, E)$. Taking $R=C(n, E)+1$ yields (4.4) for appropriate $x=x_{j}$.

Translating by $-x$, we can assume that $x=0$ in (4.4), that is $f(\Sigma) \subseteq \mathbb{R}^{n}-$ $\overline{B_{1}(0)}$. For $R:=C(n, E)+1$ with $C(n, E)$ as in (4.4), we obtain from (4.2) for all $x \in \mathbb{R}^{n}$

$$
\int_{B_{1}(x)}\left|A^{\circ}\right|^{2} \mathrm{~d} \mu \leq E / 2, \quad \text { and } \quad \int_{\mathbb{R}^{n}-B_{R}(0)}\left|A^{\circ}\right|^{2} \mathrm{~d} \mu \leq E / 2 .
$$


Now for $\tilde{f}=\phi \circ f$ where $\phi(x)=x /|x|^{2}$ we clearly have $\tilde{f}(\Sigma) \subseteq B_{1}(0)$. Moreover if $|x| \geq 1 /(2 R)$, then a ball $B_{\varrho}(x)$ of radius $\varrho=\frac{1}{2}\left(\sqrt{1+R^{-2}}-1\right)$ is mapped by $\phi^{-1}=\phi$ to a ball $B_{\varrho^{*}}\left(x^{*}\right)$ with $\varrho^{*} \leq 1$, and claim (4.1) follows from (4.7) using that the integral is locally conformally invariant. In the remaining case $|x| \leq$ $1 /(2 R)$, we use $B_{\varrho}(x) \subseteq B_{1 / R}(0)$ for $\varrho \leq 1 /(2 R)$, and obtain (4.1) from the second inequality in (4.7).

We can now prove our main theorem, recalling from (1.4) the definition of the $\omega_{p}^{n}$.

Theorem 4.2. For $n=3,4$ and $p \geq 1$, let $\mathcal{C}(n, p, \delta)$ be the class of closed, orientable, genus $p$ surfaces $f: \Sigma \rightarrow \mathbb{R}^{n}$ satisfying $\mathcal{W}(f) \leq \omega_{p}^{n}-\delta$ for some $\delta>0$. Then for any $f \in \mathcal{C}(n, p, \delta)$ there is a Möbius transformation $\phi$ and a constant curvature metric $g_{0}$, such that the metric $g$ induced by $\phi \circ f$ satisfies

$$
g=e^{2 u} g_{0} \quad \text { where } \quad \max _{\Sigma}|u| \leq C(p, \delta)<\infty .
$$

Proof. It is obviously sufficient to obtain the result for small $\delta>0$. We consider an arbitrary sequence of surfaces $f_{j} \in \mathcal{C}(n, p, \delta)$, and put $g_{j}=f_{j}^{*} g_{\text {euc }}, \mu_{j}=$ $f_{j}\left(\mu_{g_{j}}\right)$. From (1.2) we have

$$
\int_{\Sigma}\left|A_{j}^{\circ}\right|^{2} \mathrm{~d} \mu_{g_{j}} \leq 2\left(\omega_{p}^{n}-\delta\right)+8 \pi(p-1) \leq 8 \pi(p+1)-2 \delta .
$$

Using Lemma 4.1 we may assume after applying suitable Möbius transformations that

$$
f_{j}(\Sigma) \subseteq B_{1}(0) \quad \text { and } \quad \int_{B_{\varrho_{0}}(x)}\left|A_{j}^{\circ}\right|^{2} \mathrm{~d} \mu_{j} \leq 4 \pi(p+1)-\delta \text { for all } x \in \mathbb{R}^{n},
$$

where $\varrho_{0}>0$ depends only on the genus $p$. The uniformization theorem yields unique conformal metrics $e^{-2 u_{j}} g_{j}$ having the same area and constant curvature. The theorem will be proved by showing that

$$
\liminf _{j \rightarrow \infty}\left\|u_{j}\right\|_{L^{\infty}(\Sigma)}<\infty
$$

We start by recalling from (2.2) the Li-Yau-inequality

$$
\varrho^{-2} \mu_{j}\left(B_{\varrho}(x)\right) \leq C \quad \text { for all } x \in \mathbb{R}^{n}, \varrho>0
$$

For $\alpha_{j}=f_{j}\left(\mu_{g_{j}}\right.$ ᄂ|$\left.\left.A_{j}\right|^{2}\right)$ we have $\alpha_{j}\left(\mathbb{R}^{n}\right) \leq C(p)$, hence for a subsequence

$$
\mu_{j}, \alpha_{j} \rightarrow \mu, \alpha \quad \text { weakly* in } C_{c}^{0}\left(\mathbb{R}^{n}\right)^{*} .
$$

Moreover we see as in [13, page 310] that

$$
\text { spt } \mu_{j} \rightarrow \operatorname{spt} \mu \quad \text { in Hausdorff distance, }
$$


which yields further spt $\alpha \subseteq$ spt $\mu \subseteq \overline{B_{1}(0)}$. Now by Allard's integral compactness theorem for varifolds, see [12, Remark 42.8], the measure $\mu$ is an integral 2-varifold with weak mean curvature $H_{\mu} \in L^{2}(\mu)$, more precisely we have

$$
\mathcal{W}(\mu):=\frac{1}{4} \int\left|H_{\mu}\right|^{2} \mathrm{~d} \mu \leq \liminf _{j \rightarrow \infty} \mathcal{W}\left(f_{j}\right) \leq 8 \pi-\delta
$$

As discussed in the appendix of [7], the monotonicity formula from [13] applies to varifolds with weak mean curvature in $L^{2}$, in particular the Li-Yau inequality (2.3) yields

$$
\theta^{2}(\mu, x) \leq \frac{8 \pi-\delta}{4 \pi}=2-\frac{\delta}{4 \pi} \quad \text { for all } x \in \mathbb{R}^{n}
$$

We further obtain, writing $\perp$ for the projection onto $\left(T_{x} \mu\right)^{\perp}$,

$$
\int_{B_{\sigma}\left(x_{0}\right)} \frac{\left|\left(x-x_{0}\right)^{\perp}\right|^{2}}{\left|x-x_{0}\right|^{4}} \mathrm{~d} \mu(x)<\infty \quad \text { for all } x_{0} \in \mathbb{R}^{n}
$$

Let $\varepsilon_{0}=\varepsilon_{0}(n, \beta)$ be the constant from [13, Lemma 2.1]; we take $\beta=C$ for $C>0$ as in (4.11) whence $\varepsilon_{0}>0$ is universal. For $\varepsilon_{1} \in\left(0, \varepsilon_{0}\right]$ there are only finitely many points $x_{1}, \ldots, x_{K}$ with

$$
\alpha\left(\left\{x_{k}\right\}\right) \geq \varepsilon_{1}^{2} \quad \text { for } k=1, \ldots, K,
$$

in fact $K \leq C(p) \varepsilon_{1}^{-2}$. For given $\varepsilon \in\left(0, \varepsilon_{1}\right)$ we may use (4.15) and (4.14) to choose $\varrho \in\left(0, \frac{1}{2} \min _{k \neq l}\left|x_{k}-x_{l}\right|\right)$ with $\varrho<\varrho_{0}$, such that for all $k$ we have the inequalities

$$
\begin{gathered}
\alpha\left(\overline{B_{\varrho}\left(x_{k}\right)}-\left\{x_{k}\right\}\right)<\varepsilon^{2}, \\
\mu\left(\overline{B_{7 \varrho / 8}\left(x_{k}\right)}\right)<\left(2-\frac{\delta}{20}\right) \pi(7 \varrho / 8)^{2}, \\
\int_{\overline{B_{\varrho}\left(x_{k}\right)}} \frac{\left|\left(x-x_{k}\right)^{\perp}\right|^{2}}{\left|x-x_{k}\right|^{4}} \mathrm{~d} \mu(x)<\varepsilon^{2} .
\end{gathered}
$$

For any $y \notin\left\{x_{1}, \ldots, x_{K}\right\}$ there exists a radius $\varrho_{y} \in\left(0, \varrho_{0}\right)$ such that $\alpha\left(\overline{B_{\varrho_{y}}(y)}\right)<$ $\varepsilon_{1}^{2}$. Now we select finitely many points $y_{1}, \ldots, y_{L} \in \operatorname{spt} \mu-\bigcup_{k=1}^{K} B_{\varrho / 2}\left(x_{k}\right)$ such that

$$
\operatorname{spt} \mu \subseteq \bigcup_{k=1}^{K} B_{\varrho / 2}\left(x_{k}\right) \cup \bigcup_{l=1}^{L} B_{\varrho_{y_{l}} / 2}\left(y_{l}\right)
$$


By (4.12) and (4.13) we get for any $r \in(0, \varrho / 2]$ and $j$ sufficiently large (depending on $r$ )

$$
\left.\begin{array}{c}
f_{j}(\Sigma)=\operatorname{spt} \mu_{j} \subseteq \bigcup_{k=1}^{K} B_{\varrho / 2}\left(x_{k}\right) \cup \bigcup_{l=1}^{L} B_{\varrho_{y_{l}} / 2}\left(y_{l}\right), \\
\int_{B_{\varrho}\left(x_{k}\right)-B_{r}\left(x_{k}\right)}\left|A_{j}\right|^{2} \mathrm{~d} \mu_{j}<\varepsilon^{2}, \\
\int_{B_{Q_{y_{l}}}\left(y_{l}\right)}\left|A_{j}\right|^{2} \mathrm{~d} \mu_{j}<\varepsilon_{1}^{2}, \\
\mu_{j}\left(B_{7 \varrho / 8}\left(x_{k}\right)\right)<(2-\delta / 20) \pi(7 \varrho / 8)^{2}, \\
\int_{B_{\varrho}\left(x_{k}\right)-B_{r}\left(x_{k}\right)} \frac{\left|\left(x-x_{k}\right)^{\perp}\right|^{2}}{\left|x-x_{k}\right|^{4}} \mathrm{~d} \mu_{j}(x)<\varepsilon^{2},
\end{array}\right\}
$$

for $k=1, \ldots, K$ and $l=1, \ldots, L$. For the covering in (4.16) we shall now verify the assumptions of Theorem 3.1, provided that $\varepsilon_{1}=\varepsilon_{1}(n, \delta) \in\left(0, \varepsilon_{0}\right)$ and $\varepsilon=\varepsilon(n, p, \delta) \in\left(0, \varepsilon_{1}\right)$ are sufficiently small.

The condition (3.4) is clearly satisfied on the $B_{\varrho_{y_{l}}}\left(y_{l}\right), l=1, \ldots, L$, for $\varepsilon_{1}>0$ sufficiently small. For $k \in\{1, \ldots, K\}$, we have the assumptions of Lemma 2.1 and also (2.10) for any $r \in(0, \varrho / 2]$. Thus for the multiplicity $M_{k}$ as in (2.5), we get from (2.11)

$$
\begin{aligned}
(1-C \varepsilon) M_{k} \pi\left((7 \varrho / 8)^{2}-(5 r / 4)^{2}\right) & \leq \mu_{j}\left(B_{7 \varrho / 8}\left(x_{k}\right)-B_{5 r / 4}\left(x_{k}\right)\right) \\
& \leq \mu_{j}\left(B_{7 \varrho / 8}\left(x_{k}\right)\right) \\
& \leq(2-\delta / 20) \pi(7 \varrho / 8)^{2},
\end{aligned}
$$

Assuming $\varepsilon \leq \varepsilon(\delta)$ and $r / \varrho \leq c(\delta)$ this implies

$$
M_{k}=1 .
$$

For $\sigma \in\left[5 \varrho_{k} / 8,7 \varrho_{k} / 8\right]$ as in Lemma 2.1, we conclude that $f_{j}^{-1}\left(B_{\sigma}\left(x_{k}\right)\right)$ is bounded by just one circle, and can be compactified to a closed, orientable surface $\Sigma_{k}=\Sigma_{j, k}$ of genus $p_{k}=p_{j, k}$ by adding one disc. This means we have

$$
\chi\left(f_{j}^{-1}\left(B_{\sigma}\left(x_{k}\right)\right)\right)=2\left(1-p_{k}\right)-1 .
$$

As (2.6) holds with multiplicity one, the Gauß-Bonnet theorem yields

$$
\left|\int_{B_{\sigma}\left(x_{k}\right)} K_{j} \mathrm{~d} \mu_{j}+4 \pi p_{k}\right| \leq C \varepsilon^{\alpha} .
$$


Now $K_{j} \geq-\frac{1}{2}\left|A_{j}^{\circ}\right|^{2}$ by (1.1), and using $\varrho \leq \varrho_{0}$ we see from (4.8) and (4.9) that $\int_{B_{\sigma}\left(x_{k}\right)} K_{j} \mathrm{~d} \mu_{j} \geq-\frac{1}{2} \int_{B_{\ell_{k}}\left(x_{k}\right)}\left|A_{j}^{\circ}\right|^{2} \mathrm{~d} \mu_{j} \geq-\frac{1}{4} \int_{\Sigma}\left|A_{j}^{\circ}\right|^{2} \mathrm{~d} \mu_{j} \geq-2 \pi(p+1)+\frac{\delta}{2}$. In the case $p=1$ this implies the condition (3.4) with $\delta / 2$ instead of $\delta$ as well as $p_{k}=0$, for $\varepsilon \leq \varepsilon(n, \delta)$. For $p \geq 2$ we get

$$
p_{k}<(p+1) / 2<p \quad \text { for } k=1, \ldots, K .
$$

For appropriate $\left.\sigma_{k}=\sigma_{j, k} \in\right] 5 \varrho / 8,7 \varrho / 8$, we now use Lemma 2.1(b) to attach an end to the restriction of $f_{j}$ to $f_{j}^{-1}\left(B_{\sigma_{k}}\left(x_{k}\right)\right)$, obtaining an immersion $\tilde{f}_{j, k}$ : $\Sigma_{k}-\left\{q_{k}\right\} \rightarrow \mathbb{R}^{n}$ such that under $\tilde{f}_{j, k}$ a neighborhood of the puncture $q_{k}$ corresponds to a neighborhood of infinity in some affine plane, and such that

$$
\int_{\mathbb{R}^{n}-B_{\sigma_{k}}\left(x_{k}\right)}\left|\tilde{A}_{k}\right|^{2} \mathrm{~d} \tilde{\mu}_{k} \leq C \varepsilon^{2}
$$

By (4.21) and the conformal invariance of the Willmore energy, we get

$$
\mathcal{W}\left(f_{j}, B_{\sigma_{k}}\left(x_{k}\right)\right) \geq \mathcal{W}\left(\tilde{f}_{j, k}\right)-C \varepsilon^{2} \geq \beta_{p_{k}}^{n}-4 \pi-C \varepsilon^{2} .
$$

Adding $k$ discs to $\Sigma-\bigcup_{k=1}^{K} B_{\sigma_{k}}\left(x_{k}\right)$ yields a surface of some genus $p_{0}$, where

$$
\begin{aligned}
2(1-p)=\chi(\Sigma) & =\chi\left(\Sigma-\bigcup_{k=1}^{K} f_{j}^{-1}\left(B_{\sigma_{k}}\left(x_{k}\right)\right)\right)+\sum_{k=1}^{K} \chi\left(f_{j}^{-1}\left(B_{\sigma_{k}}\left(x_{k}\right)\right)\right) \\
& =2\left(1-p_{0}\right)-K+\sum_{k=1}^{K}\left(2\left(1-p_{k}\right)-1\right) \\
& =2\left(1-\sum_{k=0}^{K} p_{k}\right),
\end{aligned}
$$

which means

$$
p=\sum_{k=0}^{K} p_{k}
$$

In fact, adding the discs with bounds as in (4.21), we see that

$$
\mathcal{W}\left(f_{j}, \mathbb{R}^{n}-\bigcup_{k=1}^{K} B_{\sigma_{k}}\left(x_{k}\right)\right) \geq \beta_{p_{0}}^{n}-C(K) \varepsilon^{2} .
$$

Combining (4.22) and (4.24) implies

$\sum_{k=0}^{K}\left(\beta_{p_{k}}^{n}-4 \pi\right) \leq \mathcal{W}\left(f_{j}\right)-4 \pi+C(K) \varepsilon^{2} \leq \omega_{p}^{n}-\delta-4 \pi+C(K) \varepsilon^{2}<\omega_{p}^{n}-4 \pi$, 
if $\varepsilon \leq \varepsilon(K, \delta)$. From (4.23), (4.20) and the definition of the $\omega_{p}^{n}$, see (1.4), we now see

$$
p_{0}=p, \quad \text { and } \quad p_{k}=0 \quad \text { for } k=1, \ldots, K .
$$

Together with (4.19) this establishes (3.4) for any $p \geq 1$.

Next, claim (3.3) is immediate by taking $\varepsilon, \varepsilon_{1} \leq \varepsilon(\Lambda, \delta)$ and $r \leq \varrho / 2$ in (4.16). Moreover, for $l=1, \ldots, L$ we get from (4.16)

$$
2 \int_{B_{Q_{y_{l}}\left(y_{l}\right)}}\left|K_{j}\right| \mathrm{d} \mu_{j}, \int_{B_{Q_{y_{l}}\left(y_{l}\right)}}\left|A_{j}^{\circ}\right|^{2} \mathrm{~d} \mu_{j} \leq \int_{B_{Q_{y_{l}}}\left(y_{l}\right)}\left|A_{j}\right|^{2} \mathrm{~d} \mu_{j} \leq \varepsilon_{1}^{2},
$$

hence (3.1) and (3.2) hold for $\varepsilon_{1}>0$ small enough. For $k=1, \ldots, K$ we get from (2.6) combined with (4.17), (4.18) and (4.25), for appropriate $\sigma \in] 5 \varrho / 8,7 \varrho / 8[$,

$$
\left|\int_{B_{\sigma}\left(x_{k}\right)} K_{j} \mathrm{~d} \mu_{j}\right| \leq 2 \pi\left|\chi\left(f_{j}^{-1}\left(B_{\sigma}\left(x_{k}\right)\right)\right)-1\right|+C \varepsilon^{\alpha}=C \varepsilon^{\alpha} .
$$

From $|K| \leq \frac{1}{2}|A|^{2}=\left|A^{\circ}\right|^{2}+K$ we have the inequality

$$
\begin{aligned}
\int_{B_{\varrho}\left(x_{k}\right)}\left|K_{j}\right| \mathrm{d} \mu_{j} \leq & \frac{1}{2} \int_{B_{\varrho}\left(x_{k}\right)-B_{\varrho / 2}\left(x_{k}\right)}\left|A_{j}\right|^{2} \mathrm{~d} \mu_{j}+\int_{B_{\sigma}\left(x_{k}\right)}\left|A_{j}^{\circ}\right|^{2} \mathrm{~d} \mu_{j} \\
& +\int_{B_{\sigma}\left(x_{k}\right)} K_{j} \mathrm{~d} \mu_{j},
\end{aligned}
$$

hence we obtain from (4.16) and (4.26)

$$
\int_{B_{\varrho}\left(x_{k}\right)}\left|K_{j}\right| \mathrm{d} \mu_{j} \leq \int_{B_{\varrho}\left(x_{k}\right)}\left|A_{j}^{\circ}\right|^{2} \mathrm{~d} \mu_{j}+C \varepsilon^{\alpha} .
$$

We proceed similarly using (4.16), (4.26), (4.24), (4.25) and $\left|A^{\circ}\right|^{2}=|\vec{H}|^{2} / 2-2 K$

$$
\begin{aligned}
\frac{1}{2} \int_{B_{\ell}\left(x_{k}\right)}\left|A_{j}^{\circ}\right|^{2} \mathrm{~d} \mu_{j} & \leq \frac{1}{4} \int_{B_{\sigma}\left(x_{k}\right)}\left|\vec{H}_{j}\right|^{2} \mathrm{~d} \mu_{j}+C \varepsilon^{\alpha} \\
& \leq \mathcal{W}\left(f_{j}\right)-\mathcal{W}\left(f_{j}, \mathbb{R}^{n}-\bigcup_{k=1}^{K} B_{\sigma_{k}}\left(x_{k}\right)\right)+C \varepsilon^{\alpha} \\
& \leq \omega_{p}^{n}-\delta-\beta_{p}^{n}+C \varepsilon^{\alpha} .
\end{aligned}
$$

As $\omega_{p}^{n} \leq 8 \pi$ and $\beta_{p}^{n} \geq 4 \pi$, we conclude from (4.27)

$$
\int_{B_{\varrho}\left(x_{k}\right)}\left|K_{j}\right| \mathrm{d} \mu_{j} \leq 2\left(\omega_{p}^{n}-\beta_{p}^{n}\right)-2 \delta+C \varepsilon^{\alpha} \leq 8 \pi-2 \delta+C \varepsilon^{\alpha},
$$


which proves (3.1) taking $\varepsilon \leq \varepsilon(\delta)$. For $n=4$ we have

$$
\begin{aligned}
\int_{B_{\varrho}\left(x_{k}\right)}\left|K_{j}\right| \mathrm{d} \mu_{j}+\frac{1}{2} \int_{B_{\varrho}\left(x_{k}\right)}\left|A_{j}^{\circ}\right|^{2} \mathrm{~d} \mu_{j} & \leq \frac{3}{2} \int_{B_{\varrho}\left(x_{k}\right)}\left|A_{j}^{\circ}\right|^{2} \mathrm{~d} \mu_{j}+C \varepsilon^{\alpha} \\
& \leq 3\left(\omega_{p}^{4}-\delta-\beta_{p}^{4}\right)+C \varepsilon^{\alpha} .
\end{aligned}
$$

Now (3.2) follows by definition of $\omega_{p}^{4}$ for $\varepsilon \leq \varepsilon(\delta)$ small enough. Thus all conditions of Theorem 3.1 are verified, and application of that theorem finishes the proof.

\section{Compactness in moduli space}

The main result of this section is

Theorem 5.1. For $n \in\{3,4\}$ and $p \geq 1$, the conformal structures induced by immersions $f$ in $\mathcal{C}(n, p, \delta)$ are contained in a compact subset $K=K(p, \delta)$ of the moduli space.

The theorem follows directly from Theorem 4.2 and the following:

Lemma 5.2. Let $f: \Sigma \rightarrow \mathbb{R}^{n}$ be an immmersion of a closed, orientable surface of genus $p \geq 1$, with induced metric $g=f^{*} g_{\text {euc. Assume that }}$

$$
\mathcal{W}(f), \max _{\Sigma}|u| \leq \Lambda
$$

where $\mathcal{W}(f)$ is the Willmore energy and $g_{0}:=e^{-2 u} g$ is a conformal metric of constant curvature. Then the conformal structure induced by $g$ lies in a compact subset $K=K(n, p, \Lambda)$ of the moduli space.

Proof. We first give the proof for $p \geq 2$, where we normalize to $K_{g_{0}} \equiv-1$ by a dilation. Let $\ell>0$ be the length of a shortest closed geodesic in $\left(\Sigma, g_{0}\right)$. By the Mumford compactness theorem, see e.g. [14] Theorem C.1, the lemma follows from a lower bound for $\ell$ depending only on $n, p$ and $\Lambda$. As the hyperbolic plane has no conjugate points, we have $\operatorname{inj}\left(M, g_{0}\right)=\ell / 2$ by an argument of Klingenberg, see Lemma 4 in [5], and hyperbolic geometry implies

$$
\mu_{g_{0}}\left(B_{r}^{g_{0}}(p)\right) \geq \pi r^{2} \quad \text { for all } 0<r \leq \ell / 2 .
$$

Select a closed geodesic $\gamma$ for $g_{0}$ of length $\ell$. With respect to geodesic distance, there is a parallel neighborhood of $\gamma$ which is isometric to the quotient of $\left\{r e^{i \theta}\right.$ : $\left.r>0,|\theta-\pi / 2|<\theta_{0}\right\}$ by the action of $e^{k \ell}, k \in \mathbb{Z}$, where $\gamma$ corresponds to $\theta=\pi / 2$. Clearly $\gamma$ is not contractible since otherwise it would lift to a closed geodesic in the hyperbolic plane. By the collar lemma, see [14] Lemma D.1, we may take $\theta_{0} \in(0, \pi / 4]$ as a universal constant, as we can assume without loss of 
generality that $\ell \leq 1$. Now let $p_{1} \simeq e^{i \theta_{1}}, \ldots, p_{K} \simeq e^{i \theta_{K}}$ be a maximal collection of points with $\left|\theta_{j}-\pi / 2\right|<\theta_{0}$, such that the balls $B_{\ell}^{g_{0}}\left(p_{j}\right)$ are pairwise disjoint. By maximality the $B_{2 \ell}^{g_{0}}\left(p_{j}\right)$ cover the geodesic $\left\{e^{i \theta}:|\theta-\pi / 2|<\theta_{0}\right\}$, which implies that $K \geq c_{0} / \ell$ for a universal constant $c_{0}>0$. The closed curves $\gamma_{k}$ corresponding to $e^{t+i \theta_{k}}, 0 \leq t \leq \ell$, have length $L_{g_{0}}\left(\gamma_{k}\right) \leq C \ell$. We conclude

$$
L_{g}\left(\gamma_{k}\right) \leq C(\Lambda) L_{g_{0}}\left(\gamma_{k}\right) \leq C(\Lambda) \ell=: \varrho / 4 .
$$

Given $k \in\{1, \ldots, K\}$, we denote by $I_{k}$ the set of those $i \in\{1, \ldots, K\}$ for which $\left.f\left(p_{i}\right) \in B_{2 \varrho}\left(f\left(p_{k}\right)\right)\right\}$. For $i \in I_{k}$ and $p \in B_{\ell}^{g_{0}}\left(p_{i}\right)$ we estimate

$$
\begin{aligned}
\left|f(p)-f\left(p_{k}\right)\right| & \leq \operatorname{dist}_{g}\left(p, p_{i}\right)+\left|f\left(p_{i}\right)-f\left(p_{k}\right)\right| \leq C(\Lambda) \operatorname{dist}_{g_{0}}\left(p, p_{i}\right)+2 \varrho \\
& \leq C(\Lambda) \varrho .
\end{aligned}
$$

As the balls $B_{\ell}^{g_{0}}\left(p_{i}\right)$ are pairwise disjoint, we get putting $r=\ell / 2$ in (5.1)

$$
\left(\# I_{k}\right) \frac{\pi \ell^{2}}{4} \leq \sum_{i \in I_{k}} \mu_{g_{0}}\left(B_{\ell}^{g_{0}}\left(p_{i}\right)\right) \leq C(\Lambda) \mu_{g}\left(f^{-1} B_{C(\Lambda) \varrho}\left(f\left(p_{k}\right)\right)\right) \leq C(\Lambda) \varrho^{2},
$$

where the last step uses the Li-Yau inequality (2.2). We thus have

$$
\# I_{k} \leq C(\Lambda) \quad \text { for } k=1, \ldots, K .
$$

Now choose a maximal set $J \subseteq\{1, \ldots, K\}$ with $B_{\varrho}\left(f\left(p_{k}\right)\right) \cap B_{\varrho}\left(f\left(p_{l}\right)\right)=\emptyset$ for $k \neq l$. For any $m \in\{1, \ldots, K\}$ we have $f\left(p_{m}\right) \in B_{2 \varrho}\left(f\left(p_{k}\right)\right)$ for some $k \in J$, which means $\{1, \ldots, K\}=\bigcup_{k \in J} I_{k}$. By (5.3) this yields $K \leq \sum_{k \in J} \# I_{k} \leq$ $C(\Lambda) \# J$ and hence

$$
\# J \geq c_{0} / \ell \text { for } c_{0}=c_{0}(\Lambda)>0 .
$$

As the $B_{\varrho}\left(f\left(p_{k}\right)\right)$ are disjoint for $k \in J$, we get for some $k \in J$ using Gauß-Bonnet

$$
\int_{B_{\varrho}\left(f\left(p_{k}\right)\right)}|A|^{2} \mathrm{~d} \mu \leq \frac{1}{\# J} \int_{\Sigma}|A|^{2} \mathrm{~d} \mu \leq C(\Lambda, p) \ell .
$$

Thus for $C(\Lambda, p) \ell<\varepsilon_{0}(n, \Lambda)$ the assumptions of [13] Lemma 2.1 are satisfied, recalling also the density ratio estimate (2.2), hence there exists a $\sigma \in] \varrho / 4, \varrho / 2[$ such that $f^{-1}\left(B_{\sigma}\left(f\left(p_{k}\right)\right)\right)$ is a disjoint union of discs $D_{\sigma}^{i}, i=1, \ldots, M$. Now by (5.2) we have $L_{g}\left(\gamma_{k}\right) \leq \varrho / 4<\sigma$ which implies that $f \circ \gamma_{k}$ lies in $B_{\sigma}\left(\left(f\left(p_{k}\right)\right)\right.$, or equivalently $\gamma_{k}$ is contained in $f^{-1}\left(B_{\sigma}\left(f\left(p_{k}\right)\right)\right)$. But then $\gamma_{k}$ is actually contained in one of the discs $D_{\sigma}^{i}$, in particular $\gamma_{k}$ is contractible in $\Sigma$. But then $\gamma$ is also contractible which contradicts our previous observation.

For $p=1$ we normalize such that $\mu_{g_{0}}(\Sigma)=1$. It is well-known that $\left(\Sigma, g_{0}\right)$ is isometric to the quotient of $\mathbb{R}^{2}$ by a lattice of the form $\Gamma / \sqrt{b}$, where $\Gamma=\mathbb{Z}+$ $\mathbb{Z}(a, b)$ with $0 \leq a \leq 1 / 2, a^{2}+b^{2} \geq 1$ and $b>0$; here dilating the lattice by $1 / \sqrt{b}$ adjusts the volume to one. The length of a shortest closed geodesic is 
then $\ell=1 / \sqrt{b}$, in fact any horizontal line segment of that length corresponds to a shortest closed geodesic. We now consider points $p_{k}$ corresponding to $(0,2 k \ell)$ for $k=1, \ldots, K$. It is elementary that we can achieve $B_{\ell}^{g_{0}}\left(p_{k}\right) \cap B_{\ell}^{g_{0}}\left(p_{l}\right)=\emptyset$ where $K \geq c_{0} \ell^{-2}$. The horizontal segments yield closed geodesics $\gamma_{k}$ through $p_{k}$ of length $L_{g_{0}}\left(\gamma_{k}\right)=\ell$. From here the proof proceeds as in the case $p \geq 2$.

We finally discuss the optimality of the constants $\omega_{p}^{n}$ in Theorem 4.2. A standard example, see [13], is obtained by connecting two concentric round spheres at small distance by $p+1$ suitably scaled catenoids. This yields a sequence of embeddings $f_{j}: \Sigma \rightarrow \mathbb{R}^{3}$ of genus $p \geq 1$ with $\mathcal{W}\left(f_{j}\right) \rightarrow 8 \pi$. By a dilation we have in addition that $\mu_{g_{j}}(\Sigma)=1$ for all $j$. Assume by contradiction that there exist Möbius transformations $\phi_{j}$ and constant curvature metrics $g_{0, j}$, such that $\tilde{g}_{j}=\left(\phi_{j} \circ f_{j}\right)^{*} g_{\text {euc }}=e^{2 u_{j}} g_{0, j}$ where $\max _{\Sigma}\left|u_{j}\right|$ remain in a compact set as $j \rightarrow \infty$. Composing $\phi_{j}$ with a suitable dilation we may assume that $\mu_{\tilde{g}_{j}}(\Sigma)=1$. By Lemma 5.2, the conformal structures induced by the $g_{j}$ remain bounded, which implies that the minimal length of a noncontractible loop with respect to $g_{0, j}$, and hence with respect to $\tilde{g}_{j}$, is bounded below independent of $j$. In particular, the metric $\tilde{g}_{j}$ is not uniformly bounded by $g_{j}$ near the concentrating catenoids. Now $\phi_{j}$ is a composition of a Euclidean motion, a dilation and an inversion, hence we have

$$
\tilde{g}_{j}=c_{j}^{2} g_{j} \quad \text { or } \quad \tilde{g}_{j}=\frac{c_{j}^{2}}{\left|f_{j}-a_{j}\right|^{4}} g_{j}, \quad \text { where } c_{j}>0, a_{j} \in \mathbb{R}^{3} .
$$

In the first case, the area normalization implies $c_{j}=1$ which is a contradiction. In the second case, we note that the $a_{j}$ cannot diverge since otherwise we get for large $j$

$$
\frac{c_{j}^{2}}{16\left|a_{j}\right|^{4}} g_{j} \leq \tilde{g}_{j} \leq \frac{16 c_{j}^{2}}{\left|a_{j}\right|^{4}} g_{j} .
$$

The area normalization yields $1 / 16 \leq c_{j}^{2} /\left|a_{j}\right|^{4} \leq 16$, and we have a contradiction as before. Thus we can assume that the $a_{j}$ converge to some $a \in \mathbb{R}^{3}$, and also that the $c_{j}$ remain bounded. But since $p+1 \geq 2$ there is a catenoid concentrating at a point different to $a$, and at that point $\tilde{g}_{j}$ remains bounded by $g_{j}$. This contradiction shows that the constant $\omega_{p}^{n}$ in Theorem 4.2 cannot be replaced by a constant strictly bigger than $8 \pi$. Inverting surfaces of genus $p_{i}$ where $p_{1}+\ldots+p_{k}=p$ at points on the surface and then glueing them into a round sphere, we see similarly that $\omega_{p}^{n}$ cannot be replaced by a number bigger than $\tilde{\beta}_{p}^{n}$, and in particular that $\omega_{p}^{3}=$ $\min \left\{8 \pi, \beta_{p}^{3}\right\}$ is optimal for the statement of Theorem 4.2.

\section{Conformal parametrization}

In this section, we prove the estimate for the conformal factor needed in the proof of Theorem 3.1, thereby extending results of [10]. 
Theorem 6.1. Let $f: \mathbb{R}^{2} \rightarrow \mathbb{R}^{n}, n=3,4$, be a complete conformal immersion with induced metric $g=e^{2 u} g_{\mathrm{euc}}$ and square integrable second fundamental form satisfying

$$
\begin{aligned}
& \int_{\mathbb{R}^{2}} K \mathrm{~d} \mu_{g}=0 \quad \text { for } K=K_{g}, \\
& \int_{\mathbb{R}^{2}}|K| \mathrm{d} \mu_{g} \leq 8 \pi-\delta \quad \text { for } n=3, \\
& \int_{\mathbb{R}^{2}}|K| \mathrm{d} \mu_{g}+\frac{1}{2} \int_{\mathbb{R}^{2}}\left|A^{\circ}\right|^{2} \mathrm{~d} \mu_{g} \leq 8 \pi-\delta, \\
& \int_{\mathbb{R}^{2}}\left|A^{\circ}\right|^{2} \mathrm{~d} \mu_{g}<8 \pi,
\end{aligned}
$$

for some $\delta>0$. Then the limit $\lambda=\lim _{z \rightarrow \infty} u(z) \in \mathbb{R}$ exists, and

$$
\|u-\lambda\|_{L^{\infty}\left(\mathbb{R}^{2}\right)},\|D u\|_{L^{2}\left(\mathbb{R}^{2}\right)},\left\|D^{2} u\right\|_{L^{1}\left(\mathbb{R}^{2}\right)} \leq C(\delta) \int_{\mathbb{R}^{2}}|A|^{2} \mathrm{~d} \mu_{g} .
$$

We shall prove this theorem by constructing a solution $v: \mathbb{R}^{2} \rightarrow \mathbb{R}$ of the problem

$$
-\Delta_{g} v=K \quad \text { in } \mathbb{R}^{2}, \quad \text { and } \quad \lim _{z \rightarrow \infty} v(z)=0,
$$

which satisfies the estimates

$$
\|v\|_{L^{\infty}\left(\mathbb{R}^{2}\right)},\|D v\|_{L^{2}\left(\mathbb{R}^{2}\right)},\left\|D^{2} v\right\|_{L^{1}\left(\mathbb{R}^{2}\right)} \leq C(\delta) \int_{\mathbb{R}^{2}}|A|^{2} \mathrm{~d} \mu_{g} .
$$

The claim then follows easily. In fact, the function $u$ solves $-\Delta_{g} u=K_{g}$, see (3.5), hence the difference $u-v$ is an entire harmonic function. But [10, Theorem 4.2.1, Corollary 4.2.5], combined with (6.1), imply that $u$ is bounded. Therefore $u-v$ is also bounded and reduces to a constant $\lambda$, which proves the theorem.

Proof of Theorem 6.1 for $n=3$. The projection $\pi: S^{3} \rightarrow \mathbb{C} P^{1},\left(z_{1}, z_{2}\right) \mapsto\left[z_{1}\right.$ : $\left.z_{2}\right]$, is a Riemannian submersion for the Fubini-Study metric $g_{F S}$ on $\mathbb{C} P^{1}$. Introduce the diffeomorphism $\mathcal{P}: S^{2} \rightarrow \mathbb{C} P^{1}$ induced by composing the standard chart

$$
\psi: \mathbb{C} \rightarrow \mathbb{C} P^{1}, \psi(z)=\pi\left(\frac{(z, 1)}{\sqrt{|z|^{2}+1}}\right)
$$

with the stereographic projection

$$
T: S^{2} \backslash\left\{-e_{3}\right\} \rightarrow \mathbb{C}, T(\zeta, s)=\frac{\zeta}{1+s} .
$$

One computes $\psi^{*} g_{F S}=\left(1+|z|^{2}\right)^{-2} g_{\text {euc }}=\frac{1}{4}\left(T^{-1}\right)^{*} g_{S^{2}}$, which implies

$$
\mathcal{P}^{*} g_{F S}=\frac{1}{4} g_{S^{2}} .
$$


As the Jacobian of the normal $v:\left(\mathbb{R}^{2}, g\right) \rightarrow S^{2}$ along $f$ is $J v=|K|$, we get by (6.2)

$$
\int_{\mathbb{R}^{2}} J(\mathcal{P} \circ v) \mathrm{d} \mu_{g}=\frac{1}{4} \int_{\mathbb{R}^{2}}|K| \mathrm{d} \mu_{g} \leq 2 \pi-\delta / 4 .
$$

Recalling that the Kähler form $\omega$ on $\mathbb{C} P^{1}$, as defined in [10] 2.2, equals twice the volume form $\operatorname{vol}_{F S}$ with respect to the Fubini-Study metric, we get $\mathcal{P}^{*} \omega=$ $2 \mathcal{P}^{*} \operatorname{vol}_{F S}=\frac{1}{2} \operatorname{vol}_{S^{2}}$ by (6.7). Hence using $v^{*} \operatorname{vol}_{S^{2}}=K \operatorname{vol}_{g}=K e^{2 u}$ vol euc $_{\text {euc }}$ obtain from (6.1) that

$$
\int_{\mathbb{R}^{2}}(\mathcal{P} \circ v)^{*} \omega=\int_{\mathbb{R}^{2}}(K / 2) \operatorname{vol}_{g}=0 .
$$

We may therefore apply [10] Corollary 3.5.7 to get a solution $v: \mathbb{R}^{2} \rightarrow \mathbb{R}$ of

$$
-\Delta v=* 2(\mathcal{P} \circ v)^{*} \omega=K e^{2 u} \quad \text { on } \mathbb{R}^{2}, \quad \text { with } \quad \lim _{z \rightarrow \infty} v(z)=0,
$$

where $\Delta, *$ are taken with respect to the standard metric on $\mathbb{R}^{2}$, and such that

$$
\begin{aligned}
\|v\|_{L^{\infty}\left(\mathbb{R}^{2}\right)},\|D v\|_{L^{2}\left(\mathbb{R}^{2}\right)},\left\|D^{2} v\right\|_{L^{1}\left(\mathbb{R}^{2}\right)} & \leq C(\delta) \int_{\mathbb{R}^{2}}|D(\mathcal{P} \circ v)|^{2} \mathrm{~d} \mu_{g} \\
& =\frac{C(\delta)}{4} \int_{\mathbb{R}^{2}}|D v|^{2} \mathrm{~d} \mu_{g} \\
& =\frac{C(\delta)}{4} \int_{\mathbb{R}^{2}}|A|^{2} \mathrm{~d} \mu_{g} .
\end{aligned}
$$

As $-\Delta_{g} v=K$ by construction, the theorem follows for $n=3$.

We remark that if we use instead of $\mathcal{P}$ the map $\tilde{\mathcal{P}}$ identifying $S^{2}$ with the Graßmannian $G_{3,2} \subseteq \mathbb{C} P^{2}$, then we have $\tilde{\mathcal{P}}^{*} g_{F S}=g_{S^{2}} / 2$ instead of (6.7), which implies only

$$
\int_{\mathbb{R}^{2}} J(\tilde{\mathcal{P}} \circ v) \mathrm{d} \mu_{g}=\frac{1}{2} \int_{\mathbb{R}^{2}}|K| \mathrm{d} \mu_{g},
$$

so that instead of (6.2) we would need the stronger assumption

$$
\int_{\mathbb{R}^{2}}|K| \mathrm{d} \mu_{g} \leq 4 \pi-\delta \quad \text { for } n=3
$$

For $n \geq 4$ the Jacobian $J G$ of the Gauß map $G:(\Sigma, g) \rightarrow G_{n, 2} \subseteq \mathbb{C} P^{n-1}$ can in general not be expressed in terms of the Gauß curvature $K$ alone, more precisely it was computed in [4] that in points where $\vec{H}$ is nonzero one has

$$
J G=\frac{1}{2} \sqrt{|K|^{2}+\frac{1}{2}|\vec{H}|^{2}|B|^{2}},
$$


where $B$ is the component of $A$ orthogonal to $\vec{H}$. For the proof of Theorem 6.1 for $n=4$, we will use a correspondance $G_{4,2} \leftrightarrow S^{2} \times S^{2}$. Recall that an oriented 2-plane $P$ in $\mathbb{R}^{n}$ with oriented orthonormal basis $v, w$ is represented by

$$
[(v+i w) / \sqrt{2}] \in G_{4,2}=\left\{\left[z_{0}: \ldots: z_{n-1}\right] \mid \sum_{k=0}^{n-1} z_{k}^{2}=0\right\} \subseteq \mathbb{C} P^{n-1} .
$$

Alternatively, we can assign to $P$ the 2-vector $v \wedge w \in \Lambda_{2}\left(\mathbb{R}^{n}\right)$. For $n=4$ the Hodge operator $*: \Lambda_{2}\left(\mathbb{R}^{4}\right) \rightarrow \Lambda_{2}\left(\mathbb{R}^{4}\right)$ is an involution, that is $*^{2}=\mathrm{Id}$, and we have a direct sum decomposition $\Lambda_{2}\left(\mathbb{R}^{4}\right)=E_{+} \oplus E_{-}$into the \pm 1 eigenspaces, with corresponding projections $\Pi_{ \pm} \xi=(\xi \pm * \xi) / 2$. As the Hodge star is an isometry the decomposition is orthogonal, and both spaces $E_{ \pm}$are three-dimensional with orthonormal bases

$$
\begin{array}{ll}
e_{12}^{+}:=\left(e_{1} \wedge e_{2}+e_{3} \wedge e_{4}\right) / \sqrt{2}, & e_{12}^{-}:=\left(e_{1} \wedge e_{2}-e_{3} \wedge e_{4}\right) / \sqrt{2}, \\
e_{13}^{+}:=\left(e_{1} \wedge e_{3}+e_{4} \wedge e_{2}\right) / \sqrt{2}, & e_{13}^{-}:=\left(e_{1} \wedge e_{3}-e_{4} \wedge e_{2}\right) / \sqrt{2}, \\
e_{14}^{+}:=\left(e_{1} \wedge e_{4}+e_{2} \wedge e_{3}\right) / \sqrt{2}, & e_{14}^{-}:=\left(e_{1} \wedge e_{4}-e_{2} \wedge e_{3}\right) / \sqrt{2}
\end{array}
$$

We orient the 2-spheres $S_{ \pm}^{2}=S^{5} \cap E_{ \pm}$by selecting $e_{13}^{ \pm}, e_{14}^{ \pm}$as positive respectively negative basis for $T_{e_{12}^{ \pm}} S^{2}$. One checks that this definition is independent of the choice of a positive orthonormal basis $e_{1}, e_{2}, e_{3}, e_{4}$ for $\mathbb{R}^{4}$. Now we define $\mathcal{N}$ : $G_{4,2} \rightarrow S_{+}^{2} \times S_{-}^{2}$ by

$$
\begin{aligned}
\mathcal{N}\left(\left[\frac{v+i w}{\sqrt{2}}\right]\right) & =\sqrt{2}\left(\Pi_{+}(v \wedge w), \Pi_{-}(v \wedge w)\right) \\
& =\frac{1}{\sqrt{2}}(v \wedge w+*(v \wedge w), v \wedge w-*(v \wedge w)),
\end{aligned}
$$

and put $\mathcal{N}_{ \pm}=\Pi_{ \pm} \circ \mathcal{N}: G_{4,2} \rightarrow S_{ \pm}^{2}$. Clearly $\mathcal{N}$ is well-defined, smooth and injective.

Proposition 6.2. With respect to the Fubini-Study metric on $G_{4,2}$ and the product metric on $S_{+}^{2} \times S_{-}^{2}$, the map $\mathcal{N}: G_{4,2} \rightarrow S_{+}^{2} \times S_{-}^{2}$ defined by (6.9) is diffeomorphic and isometric up to a factor, more precisely

$$
\mathcal{N}^{*} g_{S_{+}^{2} \times S_{-}^{2}}=4 g_{F S} .
$$

Moreover, the Kähler form $\omega$ as defined in [10] has on $G_{4,2}$ the representation

$$
\omega=\left(\mathcal{N}_{+}^{*} \operatorname{vol}_{S_{+}^{2}}+\mathcal{N}_{-}^{*} \operatorname{vol}_{S_{-}^{2}}\right) / 2,
$$

where the sphere factors $S_{ \pm}^{2}$ are oriented as above. 
Proof. For an orthonormal system $v, w \in \mathbb{R}^{4}$, we put $z=(v+i w) / \sqrt{2} \in S^{7} \subseteq \mathbb{C}^{4}$ and check that $z, \bar{z}$ is a complex orthonormal system in $\mathbb{C}^{4}$. Extending $v, w$ to an orthonormal basis $v, w, \tau_{1}, \tau_{2}$ of $\mathbb{R}^{4}$, we note that $z, \bar{z}, \tau_{1}, \tau_{2} \in \mathbb{C}^{4}$ is actually a hermitian basis of $\mathbb{C}^{4}$. Now for $\alpha \in \mathbb{C}$ and $j=1,2$ we have

$$
\left.\frac{d}{d t} \sum_{k=0}^{3}\left(z+t \alpha \tau_{j}\right)_{k}^{2}\right|_{t=0}=2 \alpha \sum_{k=0}^{3} z_{k}\left(\tau_{j}\right)_{k}=0
$$

since $\bar{z}$ is perpendicular to $\tau_{j}$ in $\mathbb{C}^{4}$. Thus if $\pi: S^{7} \rightarrow \mathbb{C} P^{3}, \pi(z)=[z]$, denotes the Hopf projection, then by (6.8) we see that

$$
T_{\pi(z)} G_{4,2}=\operatorname{span}_{\mathbb{C}}\left\{D \pi(z) \tau_{1}, D \pi(z) \tau_{2}\right\} .
$$

Now $D \pi(z) i z=0$, and by definition of the Fubini-Study metric the restriction of $D \pi(z)$ to the horizontal space $\{z\}_{\mathbb{C}}^{\perp}$ is an isometry onto $T_{\pi(z)}\left(\mathbb{C} P^{3}\right)$. In particular the four vectors $D \pi(z) \tau_{j}, D \pi(z) i \tau_{j}$ for $j=1,2$ are an orthonormal basis of $T_{\pi(z)} G_{4,2}$. We calculate

$$
\begin{aligned}
D \mathcal{N}(\pi(z)) D \pi(z) \tau_{j} & =\sqrt{2} \frac{d}{d \theta}(\mathcal{N} \circ \pi) \frac{1}{\sqrt{2}}\left((\cos \theta) v+i w+(\sin \theta) \tau_{j}\right) \\
& =2 \frac{d}{d \theta}\left((\cos \theta) v+(\sin \theta) \tau_{j}\right) \wedge w \\
& =\left(\tau_{j} \wedge w+*\left(\tau_{j} \wedge w\right), \tau_{j} \wedge w-*\left(\tau_{j} \wedge w\right)\right)
\end{aligned}
$$

and

$$
\begin{aligned}
D \mathcal{N}(\pi(z)) D \pi(z) i \tau_{j} & =\sqrt{2} \frac{d}{d \theta}(\mathcal{N} \circ \pi) \frac{1}{\sqrt{2}}\left(v+i(\cos \theta) w+(\sin \theta) \tau_{j}\right) \\
& =2 \frac{d}{d \theta} v \wedge\left((\cos \theta) w+(\sin \theta) \tau_{j}\right) \\
& =\left(v \wedge \tau_{j}+*\left(v \wedge \tau_{j}\right), v \wedge \tau_{j}-*\left(v \wedge \tau_{j}\right)\right) .
\end{aligned}
$$

Writing $\left(v, w, \tau_{1}, \tau_{2}\right)=:\left(e_{1}, e_{2}, e_{3}, e_{4}\right)$ we see that $D(\mathcal{N} \circ \pi)(z)$ maps as follows:

$$
\begin{aligned}
e_{3} & \mapsto \sqrt{2}\left(-e_{14}^{+}, e_{14}^{-}\right), & & e_{4} \mapsto \sqrt{2}\left(e_{13}^{+},-e_{13}^{-}\right), \\
i e_{3} & \mapsto \sqrt{2}\left(e_{13}^{+}, e_{13}^{-}\right), & & i e_{4} \mapsto \sqrt{2}\left(e_{14}^{+}, e_{14}^{-}\right) .
\end{aligned}
$$

In particular, $D \mathcal{N}(\pi(z))$ maps an orthonormal basis of $T_{\pi(z)} G_{4,2}$ to twice an orthonormal basis of $T_{\mathcal{N}(\pi(z))}\left(S^{2} \times S^{2}\right)$, which proves (6.10). Furthermore, $\mathcal{N}$ is a local diffeomorphism by the inverse function theorem, hence $\mathcal{N}\left(G_{4,2}\right) \subseteq S^{2} \times S^{2}$ is open. As $\mathcal{N}\left(G_{4,2}\right)$ is compact, non-empty and $S^{2} \times S^{2}$ is connected, we obtain that $\mathcal{N}$ is surjective. As we already saw that $\mathcal{N}$ is injective, it is a global diffeomorphism.

The Kähler form $\omega$ on $\mathbb{C} P^{3}$ is defined in [10] by

$$
\omega(D \pi \cdot \xi, D \pi \cdot \eta)=2 g_{F S}(D \pi \cdot \xi, D \pi \cdot i \eta) \quad \text { for } \xi, \eta, \in\{z\}_{\mathbb{C}}^{\perp} .
$$


In $T_{e_{12}^{ \pm}} S_{ \pm}^{2}$, the rotation by $+\pi / 2$ is given by $J_{ \pm} e_{13}^{ \pm}= \pm e_{14}^{ \pm}$, whence

$$
\operatorname{vol}_{S_{ \pm}^{2}}(\xi, \eta)=g_{S_{ \pm}^{2}}\left(\xi, J_{ \pm} \eta\right) \quad \text { for } \xi, \eta \in T_{e_{12}^{ \pm}} S_{ \pm}^{2} .
$$

Using (6.12) we see that $D\left(\mathcal{N}_{ \pm} \circ \pi\right) \cdot i \xi=J_{ \pm} D\left(\mathcal{N}_{ \pm} \circ \pi\right) \xi$ for any $\xi \in \operatorname{span}_{\mathbb{C}}\left\{\tau_{1}, \tau_{2}\right\}$. Together with (6.10), we obtain for all $\xi, \eta \in \operatorname{span}_{\mathbb{C}}\left\{\tau_{1}, \tau_{2}\right\}$

$$
\begin{aligned}
\left(\mathcal{N}_{+}^{*} \operatorname{vol}_{S_{+}^{2}}\right. & \left.+\mathcal{N}_{-}^{*} \operatorname{vol}_{S_{-}^{2}}\right)(D \pi \cdot \xi, D \pi \cdot \eta) \\
& =\sum_{ \pm} \operatorname{vol}_{S_{ \pm}^{2}}\left(D\left(\mathcal{N}_{ \pm} \circ \pi\right) \cdot \xi, D\left(\mathcal{N}_{ \pm} \circ \pi\right) \cdot \eta\right) \\
& =\sum_{ \pm} g_{S_{ \pm}^{2}}\left(D\left(\mathcal{N}_{ \pm} \circ \pi\right) \cdot \xi, J_{ \pm} D\left(\mathcal{N}_{ \pm} \circ \pi\right) \cdot \eta\right) \\
& =\sum_{ \pm} g_{S_{ \pm}^{2}}\left(D\left(\mathcal{N}_{ \pm} \circ \pi\right) \cdot \xi, D\left(\mathcal{N}_{ \pm} \circ \pi\right) \cdot i \eta\right) \\
& =g_{S_{+}^{2} \times S_{-}^{2}}(D \mathcal{N} \cdot(D \pi \cdot \xi), D \mathcal{N} \cdot(D \pi \cdot i \eta)) \\
& =\left(\mathcal{N}^{*} g_{S_{+}^{2} \times S_{-}^{2}}\right)(D \pi \cdot \xi, D \pi \cdot i \eta) \\
& =4 g_{F S}(D \pi \cdot \xi, D \pi \cdot i \eta) \\
& =2 \omega(D \pi \cdot \xi, D \pi \cdot \eta),
\end{aligned}
$$

and (6.11) follows.

Next for any immersion $f: \mathbb{R}^{2} \rightarrow \mathbb{R}^{n}$ we introduce a modified Gauß map by

$$
\varphi:=\mathcal{N} \circ G: \mathbb{R}^{2} \rightarrow S_{+}^{2} \times S_{-}^{2},
$$

and denote by $\varphi_{ \pm}:=\Pi_{ \pm} \circ \varphi: \mathbb{R}^{2} \rightarrow S_{ \pm}^{2}$ its corresponding projections.

Proposition 6.3. The pullback of the volume form on $S_{ \pm}^{2}$ via $\varphi_{ \pm}$is given by

$$
\varphi_{ \pm}^{*} \operatorname{vol}_{S_{ \pm}^{2}}=(K \pm R) \operatorname{vol}_{g}, \quad \text { where } \quad R=2\left\langle A_{11}^{\circ} \wedge A_{12}^{\circ}, \nu_{1} \wedge v_{2}\right\rangle .
$$

Here we use an oriented orthonormal basis $e_{1}, e_{2}$ on $\left(\mathbb{R}^{2}, g\right)$, and an oriented orthonormal basis $v_{1}, v_{2}$ of normal vectors along $f$. In particular we have

$$
|R| \leq \frac{1}{2}\left|A^{\circ}\right|^{2} \quad \text { and } \quad J \varphi_{ \pm}=|K \pm R| \leq|K|+\frac{1}{2}\left|A^{\circ}\right|^{2} .
$$

Proof. We may assume that $f$ is (locally) the inclusion map, writing $e_{1,2}$ instead of $D f \cdot e_{1,2}$; also we write $e_{3,4}$ for $v_{1,2}$. It is easy to check that the definition of $R$ is independent of the choice of the (oriented) bases. We have $G=\pi\left(\left(e_{1}+i e_{2}\right) / \sqrt{2}\right)$, whence by (6.9)

$$
\varphi_{ \pm}=\sqrt{2} \Pi_{ \pm}\left(e_{1} \wedge e_{2}\right),
$$


Differentiating and using $\left\langle D_{e_{1}} e_{1}, e_{1}\right\rangle=\left\langle D_{e_{2}} e_{2}, e_{2}\right\rangle=0$ we obtain

$$
D \varphi_{ \pm} \cdot e_{k}=\sqrt{2} \Pi_{ \pm}\left(A\left(e_{1}, e_{k}\right) \wedge e_{2}+e_{1} \wedge A\left(e_{2}, e_{k}\right)\right) .
$$

Writing $A_{i j}=\alpha_{i j} e_{3}+\beta_{i j} e_{4}$ and expanding yields

$$
\begin{aligned}
D \varphi_{ \pm} \cdot e_{k} & =\sqrt{2} \Pi_{ \pm}\left(\alpha_{1 k} e_{3} \wedge e_{2}+\beta_{1 k} e_{4} \wedge e_{2}+\alpha_{2 k} e_{1} \wedge e_{3}+\beta_{2 k} e_{1} \wedge e_{4}\right) \\
& =\left(\alpha_{2 k} \pm \beta_{1 k}\right) e_{13}^{ \pm}+\left(\mp \alpha_{1 k}+\beta_{2 k}\right) e_{14}^{ \pm} .
\end{aligned}
$$

Now $e_{13}^{ \pm}, e_{14}^{ \pm}$is a positive respectively negative orthonormal basis for $T_{e_{12}^{ \pm}} S^{2}$, therefore

$$
\operatorname{det}\left(D \varphi_{ \pm}\right)= \pm \operatorname{det}\left(\begin{array}{cc}
\alpha_{21} \pm \beta_{11} & \alpha_{22} \pm \beta_{12} \\
\mp \alpha_{11}+\beta_{21} & \mp \alpha_{12}+\beta_{22}
\end{array}\right) .
$$

By choice of the bases at a point we can assume that $\alpha_{12}=0$ and $\vec{H}=H e_{3}$ for $H=$ $\alpha_{11}+\alpha_{22}$. Then $\beta_{11}=-\beta_{22}=: \beta$, and $K=\left(H^{2}-|A|^{2}\right) / 2=\alpha_{11} \alpha_{22}-\beta^{2}-\beta_{12}^{2}$. Hence

$$
\begin{aligned}
\operatorname{det}\left(D \varphi_{ \pm}\right) & =\operatorname{det}\left(\begin{array}{cc}
\beta & \pm \alpha_{22}+\beta_{12} \\
\mp \alpha_{11}+\beta_{12} & -\beta
\end{array}\right) \\
& =\alpha_{11} \alpha_{22}-\beta^{2}-\beta_{12}^{2} \pm\left(\alpha_{11}-\alpha_{22}\right) \beta_{12} \\
& =K \pm\left(\alpha_{11}-\alpha_{22}\right) \beta_{12} .
\end{aligned}
$$

On the other hand from $A_{i j}^{\circ}=A_{i j}-\frac{1}{2} \vec{H} g_{i j}$ we see that

$$
A_{11}^{\circ} \wedge A_{12}^{\circ}=\left(\left(\alpha_{11}-\frac{1}{2} H\right) e_{3}+\beta_{11} e_{4}\right) \wedge \beta_{12} e_{4}=\frac{1}{2}\left(\alpha_{11}-\alpha_{22}\right) \beta_{12} e_{3} \wedge e_{4} .
$$

This proves (6.14), and (6.15) follows easily.

Proof of Theorem 6.1 for $n=4$. We have $\left(\varphi_{+}^{*} \operatorname{vol}_{S_{+}^{2}}+\varphi_{-}^{*} \operatorname{vol}_{S_{-}^{2}}\right) / 2=K \mathrm{vol}_{g}$ from (6.14), as well as $|D \varphi|^{2}=4|D G|^{2}=2|A|^{2}$ by (6.10) and [10] 2.3. Recalling the discussion for $n=3$, it is therefore sufficient to find a solution $v: \mathbb{R}^{2} \rightarrow \mathbb{R}$ of

$$
-\Delta v=*\left(\varphi_{+}^{*} \operatorname{vol}_{S_{+}^{2}}+\varphi_{-}^{*} \operatorname{vol}_{S_{-}^{2}}\right) / 2 \text { on } \mathbb{R}^{2} \quad \text { and } \quad \lim _{z \rightarrow \infty} v(z)=0,
$$

which satisfies the estimates

$$
\|v\|_{L^{\infty}\left(\mathbb{R}^{2}\right)},\|D v\|_{L^{2}\left(\mathbb{R}^{2}\right)},\left\|D^{2} v\right\|_{L^{1}\left(\mathbb{R}^{2}\right)} \leq C(\delta) \int_{\mathbb{R}^{2}}|D \varphi|^{2} \mathrm{~d} \mathcal{L}^{2} .
$$

Using (6.14), (6.1), (6.15) and (6.3), we obtain the following estimates, assuming without loss of generality that both inequalities in (6.3) are strict,

$$
\int_{\mathbb{R}^{2}} J \varphi_{ \pm} \mathrm{d} \mu_{g}<8 \pi-\delta \quad \text { and } \quad\left|\int_{\mathbb{R}^{2}} \varphi_{ \pm}^{*} \operatorname{vol}_{S_{ \pm}^{2}}\right|<4 \pi .
$$


As explained in [10] Proposition 3.4.1, we may assume using approximation that $\varphi$ is smooth and constant outside a compact set, while keeping the assumptions (6.18). Here, we do not assume anymore that $\varphi$ is obtained as the Gauß map of some surface. Our argument will essentially follow [10] 3.4 and 3.5.

Considering $\varphi_{ \pm}$as maps from $S^{2}$ to $S_{ \pm}^{2}$ using the stereographic projection, we compute

$$
\int_{\mathbb{R}^{2}} \varphi_{ \pm}^{*} \operatorname{vol}_{S_{ \pm}^{2}}=4 \pi \operatorname{deg}\left(\varphi_{ \pm}\right) \in 4 \pi \mathbb{Z},
$$

hence we conclude from (6.18) that

$$
\operatorname{deg}\left(\varphi_{ \pm}\right)=0
$$

Defining $G=\mathcal{N}^{-1} \circ \varphi: \mathbb{R}^{2} \rightarrow G_{4,2} \subseteq \mathbb{C} P^{3}$, we get

$$
\int_{\mathbb{R}^{2}} G^{*} \omega=\int_{\mathbb{R}^{2}}\left(\varphi_{+}^{*} \operatorname{vol}_{S_{+}^{2}}+\varphi_{-}^{*} \operatorname{vol}_{S_{-}^{2}}\right) / 2=2 \pi\left(\operatorname{deg}\left(\varphi_{+}\right)+\operatorname{deg}\left(\varphi_{-}\right)\right)=0 .
$$

Let $\pi: S^{7} \rightarrow \mathbb{C} P^{3}$ be the Hopf projection. By Proposition 3.4.3 in [10] the map $G$ has a lift $F: \mathbb{R}^{2} \rightarrow S^{7}$, i.e. $G=\pi \circ F$, whose Dirichlet integral is computed as follows, using $|D G|^{2}=|D \varphi|^{2} / 4$ and $G^{*} \omega=\left(\varphi_{+}^{*} \operatorname{vol}_{S_{+}^{2}}+\varphi_{-}^{*} \operatorname{vol}_{S_{-}^{2}}\right) / 2$,

$$
4 \int_{\mathbb{R}^{2}}|D F|^{2} \mathrm{~d} \mathcal{L}^{2}=\int_{\mathbb{R}^{2}}|D \varphi|^{2} \mathrm{~d} \mathcal{L}^{2}+\left\|*\left(\varphi_{+}^{*} \operatorname{vol}_{S_{+}^{2}}+\varphi_{-}^{*} \operatorname{vol}_{S_{-}^{2}}\right)\right\|_{W^{-1,2}\left(\mathbb{R}^{2}\right)}^{2}
$$

Here for $w \in L_{l o c}^{1}\left(\mathbb{R}^{2}\right)$ the norm on the right hand side is

$$
\|w\|_{W^{-1,2}\left(\mathbb{R}^{2}\right)}=\sup \left\{\int_{\mathbb{R}^{2}} w \psi \mathrm{d} \mathcal{L}^{2}: \psi \in C_{0}^{\infty}\left(\mathbb{R}^{2}\right), \int_{\mathbb{R}^{2}}|D \psi|^{2} \mathrm{~d} \mathcal{L}^{2} \leq 1\right\} .
$$

By (6.19), the number of preimages card $\left(\varphi_{ \pm}^{-1}\{p\}\right)$ must be even for almost every $p \in S_{ \pm}^{2}$, whence (6.18) implies

$$
\operatorname{vol}_{S_{ \pm}^{2}}\left(\varphi_{ \pm}\left(\mathbb{R}^{2}\right)\right) \leq \frac{1}{2} \int_{\mathbb{R}^{2}} J \varphi_{ \pm} \mathrm{d} \mu_{g}<4 \pi-\delta / 2
$$

Therefore, we may choose open sets $U_{ \pm} \subseteq S_{ \pm}^{2}$ with $U_{ \pm} \supseteq \varphi_{ \pm}\left(\mathbb{R}^{2}\right)$ and

$$
\operatorname{vol}_{S_{ \pm}^{2}}\left(S_{ \pm}^{2}-U_{ \pm}\right) \geq \delta / 2
$$

so that $\varphi\left(\mathbb{R}^{2}\right) \subseteq U_{+} \times U_{-} \subseteq S_{+}^{2} \times S_{-}^{2}$. We shall now construct one-forms $\xi_{ \pm}$on $U_{ \pm}$with the properties

$$
d \xi_{ \pm}=\left.\operatorname{vol}_{S_{ \pm}^{2}}\right|_{U_{ \pm}} \quad \text { and } \quad\left|\xi_{ \pm}\right| \leq \frac{C}{\delta} \text { on } U_{ \pm}
$$


Using euclidean coordinates $q=(x, y, z)$, we first define a one-form $\xi_{e_{3}}$ on $S^{2}-$ $\left\{e_{3}\right\}$ by

$$
\xi_{e_{3}}=-\frac{x d y-y d x}{1-z} \quad \text { or } \quad \xi_{e_{3}}(q) \cdot v=-\frac{\left\langle e_{3} \times q, v\right\rangle}{1-\left\langle e_{3}, q\right\rangle}
$$

where $\times$ denotes the cross product. In polar coordinates $x=\sin \vartheta \cos \varphi, y=$ $\sin \vartheta \sin \varphi$ and $z=\cos \vartheta$, one readily checks that $\xi_{e_{3}}=-(1+\cos \vartheta) d \varphi$ and hence

$$
d \xi_{e_{3}}=\sin \vartheta d \vartheta \wedge \mathrm{d} \varphi=\operatorname{vol}_{S^{2}} \quad \text { on } S^{2}-\left\{e_{3}\right\},
$$

where $S^{2}$ is oriented by its exterior normal. Next for any $p \in S^{2}$ we choose $T \in$ $\mathbb{S} O$ (3) with $T p=e_{3}$ and put $\xi_{p}=T^{*} \xi_{e_{3}}$ on $S^{2}-\{p\}$. We have explicitely

$$
\xi_{p}(q) \cdot v=-\frac{\langle p \times q, v\rangle}{1-\langle p, q\rangle} \quad \text { on } S^{2}-\{p\}
$$

in particular

$$
d \xi_{p}=\operatorname{vol}_{S^{2}} \text { on } S^{2}-\{p\} \quad \text { and } \quad\left|\xi_{p}(q)\right| \leq \frac{2}{|p-q|} .
$$

For $E \subseteq S^{2}$ closed with $\operatorname{vol}_{S^{2}}(E) \geq \delta / 2$ we now define on $U=S^{2}-E$ the one-form

$$
\xi_{E}(q)=f_{E} \xi_{p}(q) \operatorname{dvol}_{S^{2}}(p),
$$

which satisfies

$$
d \xi_{E}=\left.\operatorname{vol}_{S^{2}}\right|_{U} \quad \text { and } \quad\left|\xi_{E}(q)\right| \leq \frac{2}{\delta} \int_{S^{2}} \frac{2}{|p-q|} \operatorname{dvol}_{S^{2}}(p) \leq \frac{C}{\delta} .
$$

The forms $\xi_{ \pm}$as in (6.22) are obtained by choosing orientation preserving isometries $T_{ \pm}: S_{ \pm}^{2} \rightarrow S^{2}$, and putting $\xi_{ \pm}=T_{ \pm}^{*} \xi_{E_{ \pm}}$where $E_{ \pm}=T_{ \pm}\left(S_{ \pm}^{2}-U_{ \pm}\right)$. Now define on $U_{+} \times U_{-}$the one-form $\xi=\Pi_{+}^{*} \xi_{+}+\Pi_{-}^{*} \xi_{-}$, and compute

$$
d \varphi^{*} \xi=\left.\left(\varphi_{+}^{*} \operatorname{vol}_{S_{+}^{2}}+\varphi_{-}^{*} \operatorname{vol}_{S_{-}^{2}}\right)\right|_{U_{+} \times U_{-}}, \quad \text { and } \quad\left|\varphi^{*} \xi\right| \leq \frac{C}{\delta}|D \varphi| .
$$

As $\varphi_{ \pm}\left(\mathbb{R}^{2}\right) \subseteq U_{ \pm}$, we can estimate for any $\psi \in C_{0}^{\infty}\left(\mathbb{R}^{2}\right)$

$$
\begin{aligned}
\left|\int_{\mathbb{R}^{2}} *\left(\varphi_{+}^{*} \operatorname{vol}_{S_{+}^{2}}+\varphi_{-}^{*} \operatorname{vol}_{S_{-}^{2}}\right) \psi d \mathcal{L}^{2}\right| & =\left|\int_{\mathbb{R}^{2}} d\left(\varphi^{*} \xi\right) \psi\right| \\
& =\left|\int_{\mathbb{R}^{2}}\left(\varphi_{+}^{*} \xi_{+}+\varphi_{-}^{*} \xi_{-}\right) \wedge d \psi\right| \\
& \leq \frac{C}{\delta}\|D \varphi\|_{L^{2}\left(\mathbb{R}^{2}\right)}\|D \psi\|_{L^{2}\left(\mathbb{R}^{2}\right)}
\end{aligned}
$$


hence we get by the definition of the $W^{-1,2}$ norm and by (6.20)

$$
\int_{\mathbb{R}^{2}}|D F|^{2} \mathrm{~d} \mathcal{L}^{2} \leq C(\delta) \int_{\mathbb{R}^{2}}|D \varphi|^{2} \mathrm{~d} \mathcal{L}^{2}
$$

Now (6.11) and [10, 2.2] imply that

$$
\pi^{*} \mathcal{N}^{*}\left(\Pi_{+}^{*} \operatorname{vol}_{S_{+}^{2}}+\Pi_{-}^{*} \operatorname{vol}_{S_{-}^{2}}\right) / 2=\pi^{*} \omega=\sum_{k=0}^{3} i d z_{k} \wedge d \bar{z}_{k} .
$$

From $\varphi_{ \pm}=\Pi_{ \pm} \circ \varphi$ and $\varphi=\mathcal{N} \circ \pi \circ F$ we therefore have

$$
\left(\varphi_{+}^{*} \operatorname{vol}_{S_{+}^{2}}+\varphi_{-}^{*} \operatorname{vol}_{S_{-}^{2}}\right) / 2=F^{*} \sum_{k=0}^{3} i d z_{k} \wedge d \bar{z}_{k}=2 \sum_{k=0}^{3} \operatorname{det}\left(D F_{k}\right) d x \wedge d y .
$$

As in [10, Proposition 3.3.1], we apply [2] to obtain the Hardy space estimate, combining with (6.23),

$$
\left\|*\left(\varphi_{+}^{*} \operatorname{vol}_{S_{+}^{2}}+\varphi_{-}^{*} \operatorname{vol}_{S_{-}^{2}}\right) / 2\right\|_{\mathcal{H}^{1}\left(\mathbb{R}^{2}\right)} \leq C \int_{\mathbb{R}^{2}}|D F|^{2} \mathrm{~d} \mathcal{L}^{2} \leq C(\delta) \int_{\mathbb{R}^{2}}|D \varphi|^{2} \mathrm{~d} \mathcal{L}^{2}
$$

Now [10, Theorem 3.2.1] yields the existence of a function $v: \mathbb{R}^{2} \rightarrow \mathbb{R}$ satisfying (6.16) and (6.17), thereby proving the theorem also for $n=4$.

\section{References}

[1] M. BAUER and E. KUWERT, Existence of minimizing Willmore surfaces of prescribed genus, Int. Math. Res. Not. IMRN 10 (2003), 553-576.

[2] R. Coifman, P. L. Lions, Y. Meyer and S. Semmes, Compensated compactness and Hardy spaces, J. Math. Pures Appl. 72 (1993), 247-286.

[3] D. Gilbarg and N. S. Trudinger, "Elliptic Partial Differential Equations of Second Order", third edition, Springer Verlag, Berlin - Heidelberg - New York - Tokyo, 1998.

[4] D. HofFmAnn and R. OsSERmAn, The Area of the Generalized Gaussian Image and the Stability of Minimal Surfaces in $S^{n}$ and $\mathbb{R}^{n}$, Math. Ann. 260 (1982), 437-452.

[5] W. Klingenberg, Contributions to Riemannian geometry in the large, Ann. of Math. 69 (1958), 654-666.

[6] R. KUSNER, Comparison surfaces for the Willmore problem, Pacific J. Math. 138 (1989), 317-345.

[7] E. KUWERT and R. SCHÄTZLE, Removability of point singularities of Willmore surfaces, Ann. of Math. 160 (2004), 315-357.

[8] E. KUWERT and R. SCHÄTZLE, Minimizers of the Willmore functional with precribed conformal type, preprint 2007.

[9] P. LI and S. T. YAU, A new conformal invariant and its applications to the Willmore conjecture and the first eigenvalue on compact surfaces, Invent. Math. 69 (1982), 269-291.

[10] S. MÜLlER and V. SVERAK, On surfaces of finite total curvature, J. Differential Geom. 42 (1995), 29-258. 
[11] M. SchMIDT, A proof of the Willmore conjecture, arXiv:math/0203224v2 (2002).

[12] L. Simon, "Lectures on Geometric Measure Theory", Proceedings of the Centre for Mathematical Analysis, Australian National University, Vol. 3, 1983.

[13] L. Simon, Existence of surfaces minimizing the Willmore functional, Comm. Anal. Geom. 1 (1993), 281-326.

[14] A. J. TrombA, "Teichmüller Theory in Riemannian Geometry", ETH Lectures in Mathematics, Birkhäuser, Basel-Boston-Berlin, 1992.

Mathematisches Institut

Universität Freiburg

Eckerstraße 1

D-79104 Freiburg

ernst.kuwert@math.uni-freiburg.de

Mathematisches Institut

Universität Tübingen

Auf der Morgenstelle 10

D-72076 Tübingen

rscha@everest.mathematik.uni-tuebingen.de 